\title{
Computation of $L_{\oplus}$ for several cubic Pisot numbers
}

\author{
Julien Bernat \\ Institut de Mathématiques de Luminy, UMR 6206, Campus de Luminy, Case 90\%, \\ 13288 MARSEILLE Cedex 9, FRANCE. email: bernat@iml.univ-mrs.fr
}

received 10 Jan 2005, revised 30 May 2005, accepted 29 Jun 2005.

In this article, we are dealing with $\beta$-numeration, which is a generalization of numeration in a non-integer base. We consider the class of simple Parry numbers such that $d_{\beta}(1)=0 . k_{1}^{d-1} k_{d}$ with $d \in \mathbb{N}, d \geq 2$ and $k_{1} \geq k_{d} \geq 1$. We prove that these elements define Rauzy fractals that are stable under a central symmetry. We use this result to compute, for several cases of cubic Pisot units, the maximal length among the lengths of the finite $\beta$-fractional parts of sums of two $\beta$-integers, denoted by $L_{\oplus}$. In particular, we prove that $L_{\oplus}=5$ in the Tribonacci case.

Keywords: Tribonacci number, $\beta$-numeration, $\beta$-integers, Rauzy fractal.

\section{Introduction}

This article deals with $\beta$-numeration, which is a generalization of numeration in a non-integer base. The $\beta$-numeration is born in the late 50's [35, 31]. It has known a major realization with the arrival of quasicrystals during the 80's [38], since the $\beta$-numeration allows a modelling of quasicrystals $[13,10]$. The number systems defined by the $\beta$-numeration are closely related to canonical number systems $[6,36]$, number systems generated by iterated function systems [39] or by substitutive systems of Pisot type [15]. A common feature between these fields is the property of self-similarity, which yields results in number theory [7], geometry [24], topology [8, 14], dynamical systems [40, 33, 34] and theoretical computer science [9]. Nowadays, the interactions between these domains still set up the core of the research on $\beta$-numeration. We focus with this article on applications in computer arithmetics.

Let $\beta>1$ be an algebraic integer, with $\beta \notin \mathbb{N}$. In the same way as in the case of integral bases, it is possible to expand $x \in[0,1]$ as $x=\sum_{k \in \mathbb{N}^{*}} \varepsilon_{k} \beta^{-k}$, where the sequence $\left(\varepsilon_{k}\right)_{k \in \mathbb{N}^{*}}$, called expansion of $x$ in base $\beta$, is defined on the alphabet $\mathcal{A}_{\beta}=\{0, \ldots,[\beta]\}$. Among the expansions of 1 in base $\beta$, the greatest for the lexicographic order is called $\beta$-expansion of 1 , and denoted by $d_{\beta}(1)$. This expansion is constructed by the greedy algorithm, that is, $d_{\beta}(x)=0 . \varepsilon_{1} \ldots \varepsilon_{n} \ldots$, with for all $k \in \mathbb{N}^{*}, \varepsilon_{k}=\left[\beta T_{\beta}^{k-1}(x)\right]$, where $T_{\beta}:[0,1] \longrightarrow[0,1[, x \longmapsto\{\beta x\}$. The notion of $\beta$-expansion was historically introduced by Rényi [35]. Parry produced in [31] many interesting results concerning the $\beta$-numeration, creating and studying among others a dynamical system associated to this numeration.

Let us observe that a natural expansion of 1 in base $\beta$ is 1 . However, defining $d_{\beta}(1)$ as the greatest sequence for the lexicographic order among expansions of 1 in base $\beta$ provides useful algebraic informations on $\beta$. This is why $d_{\beta}(1)$ is not defined as 1 . Another interesting expansion of 1 in base $\beta$ is the greatest sequence for the lexicographical order among those which do not end by 0 's. This expansion, denoted by $d_{\beta}^{*}(1)$, is an improper expansion of 1 in base $\beta$. At the opposite of the case of numerations in integer bases, $d_{\beta}^{*}(1)$ is worthwhile since it enables to define the Parry condition, defined below, which plays a key role in the construction and the study of the associated arithmetical language $\mathcal{L}_{\beta}$. Notably, Parry showed that for any $x \in\left[0,1\left[, d_{\beta}(x)\right.\right.$ is 1365-8050 (C) 2007 Discrete Mathematics and Theoretical Computer Science (DMTCS), Nancy, France 
the only expansion of $x$ in base $\beta$ which satisfies the following condition called the Parry condition [31]:

$$
\text { for all } n \in \mathbb{N}, S^{n}\left(\left(\varepsilon_{k}\right)_{k \in \mathbb{N}^{*}}\right)<_{l e x} d_{\beta}^{*}(1) \text {, }
$$

where $S$ is the shift map: $\mathcal{A}_{\beta}^{\mathbb{N}^{*}} \rightarrow \mathcal{A}_{\beta}^{\mathbb{N}^{*}}$ defined by $S\left(\left(\varepsilon_{k}\right)_{k \in \mathbb{N}^{*}}\right)=\left(\varepsilon_{k+1}\right)_{k \in \mathbb{N}^{*}}$. The factors of a sequence in $\mathcal{A}_{\beta}^{\mathbb{N}^{*}}$ that fulfills the Parry condition are said to be admissible, and they form the arithmetical language $\mathcal{L}_{\beta}$.

In the same way as for elements of $[0,1]$, any positive real number $x>1$ can be uniquely expanded as $x=\sum_{i=0}^{n} \varepsilon_{-i} \beta^{i}+\sum_{i \in \mathbb{N}^{*}} \varepsilon_{i} \beta^{-i}$, where $\left(\varepsilon_{i}\right)_{i \geq-n}$ satisfies the Parry condition. The part with non-negative powers of $\beta$ is then called the $\beta$-integer part of $x$, denoted by $[x]_{\beta}$; the part with negative powers of $\beta$ is called the $\beta$-fractional part of $x$, denoted by $\{x\}_{\beta}=x-[x]_{\beta}$. This allows a natural generalization for the definition of integers in base $\beta$.

Definition 0.1 The set of $\beta$-integers, denoted by $\mathbb{Z}_{\beta}$, is the set of real numbers $x$ for which there exists $n \in \mathbb{N}$ such that $x= \pm \sum_{k=0}^{n} v_{k} \beta^{k}$, where $v_{n} \ldots v_{0}$ is an admissible word.

The following set, $\operatorname{Fin}(\beta)$, is introduced in [20]. It allows to generalize the framework of numeration to the case of a non-integral base, since the elements of $\operatorname{Fin}(\beta)$ play the role of decimal numbers in base $\beta$.

Definition 0.2 The set of real numbers having a finite $\beta$-fractional part is

$$
\operatorname{Fin}(\beta)=\bigcup_{k \in \mathbb{N}} \beta^{-k} \mathbb{Z}_{\beta}
$$

Remark 1 For a given algebraic number $\beta>1$, the relation $\operatorname{Fin}(\beta)=\mathbb{Z}\left[\beta^{-1}\right]$ is known as the finiteness property, denoted by $(\mathcal{F})$. Whereas not yet fully characterized, the class of numbers satisfying the finiteness property has been extensively studied, and relations with ergodic and combinatorial properties of the associated dynamical system have been highlighted. Moreover, the finiteness property provides topologic and geometric properties for the geometrical realization of the associated dynamical system known as the Rauzy fractal, defined in Section 1.4. For instance, due to Akiyama [2], the finiteness property implies that 0 is an inner point of the Rauzy fractal $\mathcal{T}$ under the additional condition that $\beta$ is a unit. Also, Theorem 3 of the same article provides a characterization of numbers such that $\mathcal{T}$ is arcwise connected. One may refer to [1, 4, 20] for additional informations.

The sets $\mathbb{Z}_{\beta}$ and $\operatorname{Fin}(\beta)$ are not stable under usual operations like addition and multiplication. For example, when $\beta$ is the golden ratio $\frac{1+\sqrt{5}}{2}$, which is called the Fibonacci case, one checks that $\beta^{-1}+\beta^{-2}=1$. Then $d_{\beta}(1)=0.11$, and the set of admissible words consists of words defined on the alphabet $\{0,1\}$ which do not contain two consecutive occurences of 1 . The Fibonacci case is the most studied one, since $\frac{1+\sqrt{5}}{2}$ is the only Pisot number of degree two which belongs to $] 1,2[$. This means that it defines from an algebraic point of view the simplest case of numeration in a non-integer base. See for instance [26] for several properties of this numeration. It is clear that $1 \in \mathbb{Z}_{\beta}$, since $1 \in \mathcal{A}_{\beta}$ holds for any $\beta>1$. Since $1+1=2=\beta+\beta^{-2}$, one has $d_{\beta}(2)=10.01$, hence $\mathbb{Z}_{\beta}$ is not stable under addition.

Likewise, let $\beta$ be the positive root of the polynomial $X^{4}-2 X^{3}-X-1$. Then $d_{\beta}(1)=0.2011$, with $1,2 \in \mathbb{Z}_{\beta}$ and $d_{\beta}(3)=10.111(00012)^{\infty}$, hence the sum of two $\beta$-integers can have an infinite $\beta$-expansion.

In order to perform arithmetics on $\beta$-integers, say, for instance, to compute the addition of two $\beta$-integers, one must be able to renormalize expansions in base $\beta$ of real numbers obtained after adding $\beta$-integers. The renormalization step is performed by transducers, see for instance [18]. Another method consists in applying the greedy algorithm to the real number obtained after adding or multiplying $\beta$-integers, which produces its $\beta$-expansion. However, if the $\beta$-expansion of 
the sum of two $\beta$-integers is neither finite nor ultimately periodic, the required $\beta$-expansion will not be produced after a finite number of steps.

The following notations, $L_{\oplus}$ and $L_{\odot}$, are introduced in [22]. They represent the maximal possible finite length of the $\beta$-fractional parts which may appear when one adds or multiplies two $\beta$-integers. The computation of these values gives an indication on the difficulty of performing arithmetics on $\mathbb{Z}_{\beta}$.

Definition 0.3 The bound $L_{\oplus}$ is defined as $\min \left\{n \in \mathbb{N} \mid \forall x, y \in \mathbb{Z}_{\beta}, x+y \in F i n(\beta) \Rightarrow \beta^{n}(x+y) \in\right.$ $\left.\mathbb{Z}_{\beta}\right\}$ when this set is not empty, $+\infty$ otherwise.

The bound $L_{\odot}$ is defined as $\min \left\{n \in \mathbb{N} \mid \forall x, y \in \mathbb{Z}_{\beta}, x y \in F i n(\beta) \Rightarrow \beta^{n} x y \in \mathbb{Z}_{\beta}\right\}$ when this set is not empty, $+\infty$ otherwise.

Let us explain now why we are interested in the case where $L_{\oplus}$ and $L_{\odot}$ are finite. Indeed, if the sum or the product of two $\beta$-integer belongs to $\operatorname{Fin}(\beta)$, then the length of the $\beta$-fractional part of this sum or product is bounded by a constant which only depends on $\beta$. In this case, one can decide whether a given improper expansion can be renormalized into a finite or an ultimately periodic expansion, in the sense that, if during the renormalization process one gets a $\beta$-fractional part of length greater than $L_{\oplus}$, then the improper expansion corresponds to a real number that does not belong to $\operatorname{Fin}(\beta)$. Conversely, if the set of lengths of sums of two $\beta$-integers is unbounded, then performing arithmetics in $\mathbb{Z}_{\beta}$ will be very difficult if not impossible, since one cannot compute in a finite time any operation on $\beta$-integers.

It has been proven that $L_{\oplus}$ and $L_{\odot}$ are finite when $\beta$ is a Pisot number, respectively in [20] and [22]. The computation of these values is however not so easy, especially for $L_{\odot}$. The case of quadratic Pisot numbers has been studied in [13] when $\beta$ is a unit, and in [23] otherwise. However, when $\beta$ is of higher degree, it is a difficult problem to compute the exact value of $L_{\oplus}$ or $L_{\odot}$, and even to compute upper and lower bounds for these two constants. Several examples are studied in [9], where a method is described in order to compute upper bounds for $L_{\oplus}$ and $L_{\odot}$ for Pisot numbers satisfying additional algebraic properties. We prove in a forthcoming article [11] that the bounds $L_{\oplus}$ and $L_{\odot}$ are finite for the more general case of Perron numbers.

Let us detail now connections between arithmetics in base $\beta$ and the study of some mathematical structures, namely, model sets and Meyer sets, which allow a modelling of quasicrystals. For any $\beta>1, \mathbb{Z}_{\beta}$ is a discrete subset of $\mathbb{R}$. The question of finding an algebraic characterization of numbers $\beta$ such that $\mathbb{Z}_{\beta}$ is uniformly discrete is still open; however it is know that $\mathbb{Z}_{\beta}$ is uniformly discrete when $\beta$ is a Parry number. Since $\mathbb{Z}_{\beta}$ is relatively dense in $\mathbb{R}$ for all $\beta>1, \mathbb{Z}_{\beta}$ is a Delone set [25] as soon as $\mathbb{Z}_{\beta}$ is uniformly discrete. If moreover there exists $F$, a finite set, such that $\mathbb{Z}_{\beta}+\mathbb{Z}_{\beta} \subset \mathbb{Z}_{\beta}+F, \mathbb{Z}_{\beta}$ is said to be a Meyer set [29]. Such sets satisfy a strong property of regularity. A particular subset of Meyer sets is the class of model sets, which are produced by a cut-and-project scheme. Roughly speaking, these sets are obtained as the intersection of the lattice $\mathbb{Z}^{d}$ with a window, that is, a compact set of a space called internal space, that is pushed up along a half-line of another space called physical space. In the particular case of $\beta$-numeration, the window is the Rauzy fractal $\mathcal{T}$ defined in Section 1.4. See also [28, 30] for additional details.

When $\mathbb{Z}_{\beta}$ is a Meyer set, a method which enables the computation of a minimal finite set $F$ satisfying $\mathbb{Z}_{\beta}+\mathbb{Z}_{\beta} \subset \mathbb{Z}_{\beta}+F$ is defined in [5] and studied in [21], where the problem of recognizing the language which consists of sums of $\beta$-integers is studied as well. This question is related to the determination of $L_{\oplus}$, since one can choose the sets of $\beta$-fractional parts of length less than $L_{\oplus}$ for $F$ when the finiteness property $(\mathcal{F})$ holds. Note however that the set of $\beta$-fractional parts of length less than $L_{\oplus}$ is not optimal, since they may exist another set $F_{0}$ which contains less elements such that $\mathbb{Z}_{\beta}+\mathbb{Z}_{\beta} \subset \mathbb{Z}_{\beta}+F_{0}$. Let us remind that $\mathbb{Z}_{\beta}$ is a Meyer set when $\beta$ is a Pisot number [13], and that $\mathbb{Z}_{\beta}$ is a Meyer set implies that $\beta$ is either a Pisot or a Salem number [29].

The aim of this paper is, first to study a geometrical property for a particular subclass of Parry numbers, then to apply these results in order to improve the computation of an upper bound for $L_{\oplus}$. This allows us to determine the exact value of $L_{\oplus}$ for several cases of cubic Pisot numbers. This article is structured in the following way. Section 1 gathers definitions and notations that are 
introduced in the framework of $\beta$-numeration. Section 2 is devoted to the class of $\beta$ which satisfy the following relation:

$$
\text { there exist } d \geq 2, k_{1}, k_{d} \in \mathbb{N}, k_{1} \geq k_{d} \text { such that } d_{\beta}(1)=0 . k_{1}^{d-1} k_{d} .
$$

Such numbers define confluent numeration systems, introduced and studied by Frougny [17]. This is why we will refer to confluent Parry numbers for any Parry number satisfying (1) from now on. We find the following geometric interpretation for confluent Parry numbers.

Proposition 2.5. Let $\beta$ be a confluent Parry number. There exists $\vec{C} \in \mathbb{R}^{d-1}$ such that the Rauzy fractal $\mathcal{T}$ is stable under the central symmetry $S_{\vec{C}}: \mathbb{R}^{d-1} \rightarrow \mathbb{R}^{d-1}, \vec{X} \longmapsto 2 \vec{C}-\vec{X}$.

For a given Pisot number $\beta$, we introduce and study a symmetric centered set $\mathcal{T}^{\prime}$, which contains the Rauzy fractal $\mathcal{T}$. The equality $\mathcal{T}^{\prime}=\mathcal{T}$ holds exactly when $\beta$ is a confluent Parry number. The study of $\mathcal{T}^{\prime}$ in Section 3.1 provides inequalities satisfied by any Galois conjugate of $\beta$ (Propositions $3.4,3.5)$. This allows us to improve a method detailed in [9], which enables the computation of upper and lower bounds for $L_{\oplus}$ under an algebraic condition on $\beta$. In particular, we determine the exact value of $L_{\oplus}$ for several cases of cubic Pisot units in Section 3.2. Finally, we focus in Section 3.3 on the Tribonacci case, that is, when $\beta$ is the positive root of the polynomial $X^{3}-X^{2}-X-1$. We improve by the following theorem the result $5 \leq L_{\oplus} \leq 6[9]$.

Theorem 3.9. When $d_{\beta}(1)=0.111$, we have $L_{\oplus}=5$.

\section{Definitions and notation}

We refer mainly for the notation introduced here to [32] and [26].

\subsection{Substitutions}

Let $\mathcal{A}$ be a finite set that is called alphabet. Endowed with the concatenation, $\mathcal{A}$ generates a monoid called set of finite words, denoted by $\mathcal{A}^{*}$. Let $\varepsilon$ denote the empty word. When $\mathcal{A}$ is a totally ordered set, the sets $\mathcal{A}^{\mathbb{Z}}, \mathcal{A}^{\mathbb{N}}$ and $\mathcal{A}^{*}$ are endowed with the lexicographic order $<_{\text {lex }}$.

Let $v=v_{1} \ldots v_{n} \in \mathcal{A}^{*}$. The number of letters of $v$, called length of $v$, is denoted by $|v|$; the number of occurrences of $a \in \mathcal{A}$ in $v$ is denoted by $|v|_{a}$. A word $w$ is called factor of $v$ if there exists $p, s \in \mathcal{A}^{*}$ such that $v=p w s$. The mirror image of $v=v_{1} \ldots v_{n}$ is defined as $\widetilde{v}=v_{n} \ldots v_{1}$, and the mirror image map as the map $\mathcal{A}^{*} \rightarrow \mathcal{A}^{*}, u \longmapsto \tilde{u}$. A word $v \in \mathcal{A}^{*}$ is said to be a palindrome when $\widetilde{v}=v$. When $v \in \mathcal{A}^{\mathbb{N}}$, we set $|v|=+\infty$; a word $w$ is said to be a factor of $v$ if there exists $v^{\prime}$, a prefix of $v$, such that $w$ is a factor of $v^{\prime}$.

Let $\mathcal{A}=\left\{a_{1}, \ldots, a_{d}\right\}$. Let $\left(\overrightarrow{e_{j}}\right)_{j \in \llbracket 1, \ldots, d \rrbracket}$ be the canonical basis of $\mathbb{Z}^{d}$. The morphism $f: \mathcal{A}^{*} \rightarrow \mathbb{Z}^{d}$ that fulfills $f\left(a_{j}\right)=\overrightarrow{e_{j}}$ for all $j \in \llbracket 1, \ldots, d \rrbracket$ is called the abelianization homomorphism.

Any map $\sigma: \mathcal{A} \rightarrow \mathcal{A}^{*}$, extended to a morphism on $\mathcal{A}^{*}$ by concatenation, is called substitution. The substitution $\sigma$ is said non-erasing if for every $a \in \mathcal{A}, \sigma(a) \neq \varepsilon$. Let $\sigma$ be a substitution defined on the alphabet $\mathcal{A}$. The incidence matrix of $\sigma$ is defined as the square matrix $M_{\sigma} \in \mathcal{M}_{d}(\mathbb{N})$ such that, for every $(i, j) \in \llbracket 1, \ldots, d \rrbracket^{2}, M_{\sigma}[i, j]=\left|\sigma\left(a_{j}\right)\right|_{a_{i}}$. The substitution is primitive if there exists $n \in \mathbb{N}$ such that for every $(i, j) \in \llbracket 1, \ldots, d \rrbracket^{2}, M_{\sigma}^{n}[i, j] \geq 1$. The substitution is said to be unimodular if $\left|\operatorname{det} M_{\sigma}\right|=1$. The substitution is said to be of Pisot type if the eigenvalues of the incidence matrix $M_{\sigma}$ satisfy the following: there exists a dominant eigenvalue $\beta>1$ such that for every other eigenvalue $\alpha$, one has $0<|\alpha|<1$. Note that the characteristic polynomial of $M_{\sigma}$ is irreducible when $\sigma$ is of Pisot type. Let us remind that a Pisot number is an algebraic integer whose any Galois conjugate $\alpha \neq \beta$ satisfies $|\alpha|<1$, and that the dominant eigenvalue of $M_{\sigma}$ is a Pisot numbers when $\sigma$ is of Pisot type.

Let $\sigma$ be a substitution. If there exists $k \in \mathbb{N}^{*}$ such that $\sigma^{k}(\omega)=\omega$, we say that $\omega$ is a periodic point of $\sigma$. In particular, when $\sigma(\omega)=\omega, \omega$ is said to be a fixed point of $\sigma$. When $\sigma$ is primitive, we denote by $\mathcal{L}_{\sigma}$ the language generated by the substitution, that is, $\mathcal{L}_{\sigma}=\bigcup_{k \in \mathbb{N}}\left\{\right.$ factors of $\left.\sigma^{k}(a)\right\}$, where $a \in \mathcal{A}$. Note that, since we assume that $\sigma$ is primitive, the definition of $\mathcal{L}_{\sigma}$ does not depend on the choice of $a$. 


\subsection{Introduction to $\beta$-numeration}

Let $\beta>1$ be an algebraic integer. The set $\mathbb{N} \cap\left[0, \beta\left[\right.\right.$ is called $\beta$-alphabet and denoted by $\mathcal{A}_{\beta}$. Let $x \in[0,1]$. Any sequence $\left(\varepsilon_{k}\right)_{k \in \mathbb{N}^{*}} \in \mathcal{A}_{\beta}^{\mathbb{N}^{*}}$ satisfying $x=\sum_{k \in \mathbb{N}^{*}} \varepsilon_{k} \beta^{-k}$ is called expansion of $x$ in base $\beta$. The notion of expansion of $x$ in base $\beta$ is extended on $\mathbb{R}_{+}$by considering sequences of $\mathcal{A}_{\beta}^{\mathbb{Z}}$. Among the expansions of $x$ in base $\beta$, the greatest for the lexicographical order is called $\beta$-expansion of $x$. This expansion, denoted by $d_{\beta}(x)=0 . \varepsilon_{1} \ldots$, is constructed by the greedy algorithm, that is, $\varepsilon_{k}=\left[\beta T_{\beta}^{k-1}(x)\right]$ for all $k \in \mathbb{N}^{*}$, where $T_{\beta}:\left[0,1\left[\rightarrow\left[0,1\left[, y \longmapsto\{\beta y\}\right.\right.\right.\right.$. Let $d_{\beta}$ denote the map: $[0,1] \rightarrow \mathcal{A}_{\beta}^{\mathbb{N}^{*}}, x \longmapsto\left(\varepsilon_{i}\right)_{i \in \mathbb{N}^{*}}$. We recall that the map $d_{\beta}$ is increasing if $\mathcal{A}_{\beta}^{\mathbb{N}^{*}}$ is endowed with the lexicographic order.

Parry showed in [31] that, for any $x \in\left[0,1\left[, d_{\beta}(x)\right.\right.$ is the unique expansion of $x$ in base $\beta$ which satisfy the following relation called Parry condition:

$$
\text { for all } n \in \mathbb{N}, S^{n}(v)<_{\text {lex }} d_{\beta}^{*}(1) .
$$

Any word or sequence $v \in \mathcal{A}_{\beta}^{*} \cup \mathcal{A}_{\beta}^{\mathbb{N}}$ which satisfy (2) is said to be admissible. Let $\mathcal{L}_{\beta}$ denote the set of admissible words. The set of non-negative $\beta$-integers, denoted by $\mathbb{Z}_{\beta}^{+}$, is defined as the set of non-negative real numbers $x$ satisfying $x=\sum_{k=0}^{n} v_{i} \beta^{i}$, where $v_{n} \ldots v_{0} \in \mathcal{L}_{\beta}$. The set of $\beta$-integers is defined as $\mathbb{Z}_{\beta}= \pm \mathbb{Z}_{\beta}^{+}$. Since $\mathbb{Z}_{\beta}^{+}$is a discrete set, one may define for $x \in \mathbb{R}_{+}$the $\beta$-integer part of $x$ as $[x]_{\beta}=\max _{p \in \mathbb{Z}_{\beta}^{+}}\{p \leq x\}$, and the $\beta$-fractional part of $x$ as $\{x\}_{\beta}=x-[x]_{\beta}$. For $x>1$, we set $d_{\beta}(x)=v . w$, where $v$ and $w$ respectively are the unique expansions of $[x]_{\beta}$ and $\{x\}_{\beta}$ in base $\beta$ which satisfy the Parry condition.

For $x \in \mathbb{R}_{+}$, let $l_{i}(x)$ denote the length of the $\beta$-integer part of $x$, that is, $l_{i}(x)=N+1$, where $N \in \mathbb{N}$ satisfies $\beta^{N} \leq x<\beta^{N+1}$ when $x \geq 1,0$ otherwise. If there exists $M \in \mathbb{N}$ such that $\varepsilon_{M} \neq 0$ and $\varepsilon_{n}=0$ for all $n>M$, we say that the $\beta$-expansion of $x$ is finite. In this case, the ending consecutive occurences of the letter 0 are omitted; $d_{\beta}(x)=\varepsilon_{N} \ldots \varepsilon_{0} \cdot \varepsilon_{-1} \ldots \varepsilon_{-M}$, and we set $l_{f}(x)=M$, which denotes the length of the $\beta$-fractional part of $x$. The set of non-negative real numbers having a finite $\beta$-expansion is denoted by $\operatorname{Fin}(\beta)^{+}$, and we set $\operatorname{Fin}(\beta)= \pm \operatorname{Fin}(\beta)^{+}$.

When $d_{\beta}(1)$ is either finite or ultimately periodic, $\beta$ is said to be a Parry number. More precisely, $\beta$ is said to be a sofic Parry number when $d_{\beta}(1)$ is ultimately periodic, and a simple Parry number when $d_{\beta}(1)$ is finite. The notion of Parry number was historically introduced by Parry [31] as $\beta$-number. Bertrand [12] and Schmidt [37] have independently proven that Pisot numbers are Parry numbers. When $\beta$ is a simple Parry number, we denote by $m$ the length of $d_{\beta}(1)$. In the sofic Parry case, we respectively denote by $n$ and $p$ the lengths of the preperiod and of the period of $d_{\beta}(1)$, and we set $m=n+p$ for convenience. More details about Parry numbers can be found in $[26]$.

Remark 2 One easily checks that $m \geq d$, where $d$ is the algebraic degree of $\beta$. There exist Parry numbers such that $m>d$; for instance, the smallest Parry number is the positive root of the polynomial $X^{3}-X-1$, for which one has $d_{\beta}(1)=0.10001$.

Let $\beta$ be an algebraic integer such that there exists a Galois conjugate $\alpha$ of $\beta$ which satisfies $|\alpha|<1$. Let $z \in \mathbb{C}$. We call $\alpha$-expansion of $z$ any admissible sequence $\left(\varepsilon_{k}\right)_{k \in \mathbb{Z}}$ such that $z=$ $\sum_{k \in \mathbb{Z}} \varepsilon_{k} \alpha^{-k}$.

Remark 3 There exist complex numbers having multiple $\alpha$-expansions. For instance, consider the Fibonacci case, for which the Galois conjugate of $\beta$ is $\alpha=\frac{1-\sqrt{5}}{2}$. Let the admissible sequences $\left(\varepsilon_{k}\right)_{k \in \mathbb{Z}}$ and $\left(\delta_{k}\right)_{k \in \mathbb{Z}}$ be defined by $\varepsilon_{j}=1$ when $j \in 2 \mathbb{N}+1, \varepsilon_{j}=0$ otherwise; $\delta_{j}=1$ when $j \in\{-1\} \cup 2 \mathbb{N}^{*}, \delta_{j}=0$ otherwise. Then $-1=\sum_{k \in \mathbb{Z}} \varepsilon_{k} \beta^{-k}=\sum_{k \in \mathbb{Z}} \delta_{k} \beta^{-k}$. Hence the real number -1 admits both $\left(\varepsilon_{k}\right)_{k \in \mathbb{Z}}$ and $\left(\delta_{k}\right)_{k \in \mathbb{Z}}$ as $\alpha$-expansions. 


\subsection{Notion of $\beta$-substitution}

For any Parry number $\beta$, it is possible to define a substitution which is associated to $\beta$ in a canonical way. Let $\beta$ be a simple (resp. sofic) Parry number, with $d_{\beta}(1)=0 . k_{1} \ldots k_{m}$ (resp. $\left.d_{\beta}(1)=0 . k_{1} \ldots k_{n}\left(k_{n+1} \ldots k_{n+p}\right)^{\infty}\right)$. We remind that the elements of $\left(k_{j}\right)_{j \in \llbracket 1, \ldots, m \rrbracket}$ are nonnegative integers, and that for all $n \in \mathbb{N}^{*}, S^{n}\left(d_{\beta}(1)\right)<_{l e x} d_{\beta}(1)$. The substitution $\sigma_{\beta}$ is defined on $\left\{a_{1}, \ldots, a_{m}\right\}$ by:

1. for all $j \in \llbracket 1 \ldots m-1 \rrbracket, \sigma_{\beta}\left(a_{j}\right)=a_{1}^{k_{j}} a_{j+1}$,

2. $\sigma_{\beta}\left(a_{m}\right)=a_{1}^{k_{m}}$ in the simple Parry case, $\sigma_{\beta}\left(a_{m}\right)=a_{1}^{k_{m}} a_{n+1}$ otherwise.

The substitution associated in this way to a simple (sofic) Parry number $\beta$ is called a simple (sofic) $\beta$-substitution, and is denoted by $\sigma_{\beta}$. Note that a $\beta$-substitution is primitive, admits a unique fixed point $\omega_{\beta}$ and is defined on an alphabet containing at least two letters. In particular, the set of the factors of $\omega_{\beta}$ defines a language denoted by $\mathcal{L}_{\sigma}$. See [16, 41], where these substitutions are introduced, for more details.

When $\beta$ is a simple (sofic) Parry number, the incidence matrix $M_{\sigma}$ of $\sigma$ is

$$
\left(\begin{array}{cccccc}
k_{1} & \ldots & \ldots & \ldots & \ldots & k_{m} \\
1 & 0 & \ldots & \ldots & \ldots & 0 \\
0 & \ddots & \ddots & & & \vdots \\
\vdots & \ddots & \ddots & \ddots & & 0 \\
\vdots & & \ddots & \ddots & \ddots & \vdots \\
0 & \ldots & \ldots & 0 & 1 & 0
\end{array}\right) \text { (simple case) or }\left(\begin{array}{cccccc}
k_{1} & \ldots & \ldots & \ldots & \ldots & k_{n+p} \\
1 & 0 & \ldots & \ldots & \ldots & 0 \\
0 & \ddots & \ddots & & & \vdots \\
\vdots & \ddots & \ddots & \ddots & & 1 \\
\vdots & & \ddots & \ddots & \ddots & \vdots \\
0 & \ldots & \ldots & 0 & 1 & 0
\end{array}\right) \text { (sofic case). }
$$

Remark 4 Let us recall that, if $\sigma_{\beta}$ is of Pisot type, then $\beta$ is a Pisot number, but the converse is false. For example, the positive root $\beta$ of the polynomial $X^{3}-X-1$ is a Pisot number, whereas $\sigma_{\beta}$ is not of Pisot type, since the characteristic polynomial of the incidence matrix is $X^{5}-X^{4}-1=\left(X^{3}-X-1\right)\left(X^{2}-X+1\right)$, which admits two roots of modulus 1 .

\subsection{Rauzy fractals}

Rauzy fractals were first introduced by Rauzy in [33, 34], then by Thurston in [41]. They provide a geometrical representation of the dynamical system defined by the associated $\beta$-substitution. In the framework of numeration, they are called central tiles by Akiyama $[2,3,4]$.

Let $\beta$ be a Pisot number of degree $d$, and $\left\{\alpha_{j}\right\}_{j \in \llbracket 1, \ldots, r+s \rrbracket}$ be the set of the Galois conjugates which differ from $\beta$ and which have a non-negative imaginary part. Let $\mu_{\beta}(X)=X^{d}-\sum_{j=1}^{d} b_{j} X^{d-j}$ denote the minimal polynomial of $\beta$ on $\mathbb{Z}$, which admits $r+1$ real roots and $2 s$ complex roots, hence $d-1=r+2 s$. For convenience, we set $J=\llbracket 1, \ldots, r+s \rrbracket$.

Let $M_{\beta}$ be the companion matrix of $\mu_{\beta}$, defined as follows:

$$
M_{\beta}=\left(\begin{array}{ccccc}
b_{1} & \ldots & \ldots & \ldots & b_{d} \\
1 & 0 & \ldots & \ldots & 0 \\
0 & \ddots & \ddots & & \vdots \\
\vdots & \ddots & \ddots & \ddots & \vdots \\
0 & \ldots & 0 & 1 & 0
\end{array}\right)
$$

For all $j \in J$, let $\mathcal{H}_{j}$ denote the eigenspace associated to the eigenvalue $\alpha_{j}$, and let $\mathcal{D}$ denote the eigenspace associated to the dominant eigenvalue $\beta$. Note that $\mathcal{H}_{j} \simeq \mathbb{R}$ when $\alpha_{j} \in \mathbb{R}, \mathcal{H}_{j} \simeq \mathbb{C}$ otherwise. It is possible to describe $\mathbb{R}^{d}$ as $\mathcal{D} \oplus \mathcal{H}$, where $\mathcal{H}$ denotes the direct sum of the stable 
sub-spaces $\left\{\mathcal{H}_{j}\right\}_{j \in J}$. Moreover, there exists $\vec{w} \in \mathcal{D}$ and a base of $\mathcal{H}=\underset{j \in J}{\oplus} \mathcal{H}_{j}$ constituted by $r+2 s$ complex eigenvectors $\left(\overrightarrow{v_{j}}\right)_{j \in \llbracket 1, \ldots, r+2 s \rrbracket}$, which can be chosen such that the following equalities hold:

$$
\begin{gathered}
\overrightarrow{e_{1}}=\sum_{j \in J} \overrightarrow{v_{j}}+\vec{w}, \\
\forall j \in \llbracket 1, \ldots, s \rrbracket, \forall i \in \llbracket 1, \ldots, d \rrbracket, v_{r+j}[i]=\overline{v_{r+s+j}[i]},
\end{gathered}
$$

where $v_{k}[i]$ denotes the $i$-th coordinate of $v_{k} \in \mathbb{R}^{r} \times \mathbb{C}^{d-r}$ and $z \longmapsto \bar{z}$ the standard conjugacy on $\mathbb{C}$. These relations allow us to define $\overrightarrow{p_{\mathcal{D}}}$ and, for all $j \in J, \overrightarrow{p_{\mathcal{H}}}$, which are the projections on each associated subspace along the direct sum of the others. We then set $p_{\mathcal{D}}$ and, for all $j \in J, p_{\mathcal{H}_{j}}$ as the coordinate maps in the base $\left(\vec{w}, \overrightarrow{v_{1}}, \ldots, \overrightarrow{v_{r+2 s}}\right)$ of the associated projection maps, that is, one gets for all $\vec{X} \in \mathbb{R}^{d}$ :

$$
\begin{gathered}
\overrightarrow{p_{\mathcal{H}_{j}}}(\vec{X})=p_{\mathcal{H}_{j}}(\vec{X}) \overrightarrow{v_{j}} \text {, for all } j \in \llbracket 1, \ldots, r \rrbracket, \\
\overrightarrow{p_{\mathcal{H}_{j}}}(\vec{X})=p_{\mathcal{H}_{j}}(\vec{X}) \operatorname{Re}\left(\overrightarrow{v_{j}}\right) \text {, for all } j \in \llbracket r+1, \ldots, r+s \rrbracket .
\end{gathered}
$$

For all $j \in J$, we define $\tau_{j}$ as the field morphism: $\mathbb{Q}(\beta) \rightarrow \mathbb{Q}\left(\alpha_{j}\right), \beta \longmapsto \alpha_{j}$. We denote by $\tau$ the map:

$$
\tau: \mathbb{Q}(\beta) \rightarrow \mathbb{R}^{r} \times \mathbb{C}^{s} \simeq \mathbb{R}^{d-1}, x \longmapsto\left(\tau_{1}(x), \ldots, \tau_{r+s}(x)\right) .
$$

Definition 1.1 The set $\overline{\left\{\tau(x), x \in \mathbb{Z}_{\beta}^{+}\right\}}$is called Rauzy fractal or central tile, denoted by $\mathcal{T}$.

Note that a Rauzy fractal is a compact set by definition, since it is a bounded set.

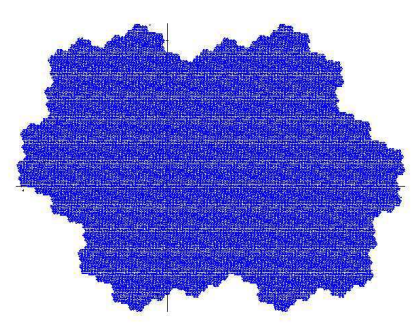

Fig. 1: Rauzy fractal $\mathcal{T}$ defined by $d_{\beta}(1)=0.111$

Example 1 Figure 1 shows the Rauzy fractal associated to the Tribonacci case, defined by $d_{\beta}(1)=$ 0.111 .

\section{Rauzy fractals and symmetric sets}

In this section, we introduce a geometric representation set $\mathcal{T}^{\prime}$ for the numeration defined by $\beta$, a given algebraic number. The set $\mathcal{T}^{\prime}$, which admits a symmetric property that we explain later, contains the Rauzy fractal $\mathcal{T}$. Then, following [9], the study of $\mathcal{T}^{\prime}$ provides inequalities satisfied by the images under $\tau_{j}$ of the finite $\beta$-fractional parts of sums of two $\beta$-integers. This allows us to define an algorithm which improves the determination of an upper bound and of a lower bound for $L_{\oplus}$.

\subsection{Set of $\beta$-integers and admissibility}

The set of non-negative $\beta$-integers is defined as

$$
\mathbb{Z}_{\beta}^{+}=\bigcup_{n \in \mathbb{N}}\left\{\sum_{k=0}^{n} v_{k} \beta^{k}, v_{n} \ldots v_{0} \in \mathcal{L}_{\beta}\right\} .
$$


When one performs arithmetics on $\beta$-integers, the computation of the admissibility condition is not so easy, and the renormalization process significantly slows down any algorithmic process. If we remove the admissibility condition, we obtain a set that contains $\mathbb{Z}_{\beta}^{+}$, which is

$$
\Sigma_{\beta}^{+}=\bigcup_{n \in \mathbb{N}}\left\{\sum_{k=0}^{n} v_{k} \beta^{k}, v_{n} \ldots v_{0} \in \mathcal{A}_{\beta}^{n+1}\right\} .
$$

Note that, for any $x \in \mathbb{Z}_{\beta}^{+}$, there exists a unique admissible word $v_{n} \ldots v_{0} \in \mathcal{L}_{\beta}$ such that $x=$ $\sum_{k=0}^{n} v_{k} \beta^{k}$, whereas there may exist $x \in \Sigma_{\beta}, n \in \mathbb{N}$ and two distincts words $v, w \in \mathcal{A}_{\beta}^{*}$ such that $x=\sum_{k=0}^{n} v_{k} \beta^{k}=\sum_{k=0}^{n} w_{k} \beta^{k}$. As an example, the words 100 and 011 represent the same real number in Fibonacci base, since $\beta^{2}=\beta+1$. The word 011 is defined on $\mathcal{A}_{\beta}^{*}$, but it does not belong to $\mathcal{L}_{\beta}$. Hence, if $\beta$ is such that $\mathbb{Z}_{\beta}^{+}=\Sigma_{\beta}^{+}$, we can omit the admissibility condition in order to generate the set of $\beta$-integers.

The set of Parry numbers for which $\mathbb{Z}_{\beta}^{+}=\Sigma_{\beta}^{+}$is exactly the set of numbers which define confluent numeration systems, which were introduced and studied by Frougny [17]. Confluent numeration systems characterize numeration systems for which any finite expansion on the alphabet $\mathcal{A}_{\beta}$ can be renormalized as an admissible word which represent a $\beta$-integer. Therefore confluent Parry numbers, that is, Parry numbers which define a confluent numeration system, are the numbers for which there is no carry propagating to the right. We will use later the following proposition, which gathers different results from [19] and [17].

Proposition 2.1 [19, 17] Let $\beta$ be a Parry number. The following assertions are equivalent.

1. One has $d_{\beta}(1)=0 . k_{1}^{d-1} k_{d}$, with $k_{1}, k_{d} \in \mathbb{N}$ and $k_{1} \geq k_{d}$,

2. the language $\mathcal{L}_{\sigma}$ is stable under mirror image,

3. there exist infinitely many prefixes of $\omega$, the fixed point of $\sigma_{\beta}$, that are palindromes,

4. one has $\mathbb{Z}_{\beta}^{+}=\Sigma_{\beta}^{+}$.

When any of these assertions holds, $\beta$ is said to be a confluent Parry number.

It is proved in $[20]$ that confluent Parry numbers are Pisot numbers such that $\operatorname{Fin}(\beta)=\mathbb{Z}\left[\beta^{-1}\right]$, that is, the finiteness property holds true. Moreover, the class of confluent Parry numbers defines a class of $\beta$-substitutions whose associated dynamical system has purely discrete spectrum [40], and the complexity of their substitutive language is affine [19].

\subsection{The symmetric property for confluent Parry numbers}

We focus on a geometric property for the Rauzy fractal $\mathcal{T}$ defined by a Pisot number $\beta$, indeed, we see in this section that $\overline{\tau\left(\Sigma_{\beta}^{+}\right)}$is stable under a central symmetry map defined below. Note that, since $\mathbb{Z}_{\beta}^{+} \subset \Sigma_{\beta}^{+}$, we get $\mathcal{T} \subset \overline{\tau\left(\Sigma_{\beta}^{+}\right)}$. Moreover, according to Proposition 2.1, the set of confluent Parry number characterizes the Parry numbers for which $\mathcal{T}=\overline{\tau\left(\Sigma_{\beta}^{+}\right)}$.

We will see in Section 3.1 that the relations developed here enable a covering of $\mathcal{T}$ with balls having arbitrarily small positive radius, in a more accurate way than in [9]. In particular, this covering is accurate enough to allow the computation, in several cases of cubic Pisot numbers, of the maximal length of the finite $\beta$-fractional parts for the sums of two $\beta$-integers.

Definition 2.2 Let $d \in \mathbb{N}^{*}$, and let $\mathcal{E}$ be a bounded set of $\mathbb{R}^{r} \times \mathbb{C}^{s} \simeq \mathbb{R}^{d-1}$. The set $\mathcal{E}$ is said to be centrally symmetric if there exists $\vec{C} \in \mathbb{R}^{r} \times \mathbb{C}^{s}$ such that $\mathcal{E}$ is stable under the symmetry $S_{\vec{C}}: \mathbb{R}^{r} \times \mathbb{C}^{s} \rightarrow \mathbb{R}^{r} \times \mathbb{C}^{s}, \vec{X} \longmapsto 2 \vec{C}-\vec{X}$. In this case, $\vec{C}$ is called center of the set $\mathcal{E}$. 
Lemma 2.3 Let $\beta$ be a Pisot number. Then $\overline{\tau\left(\Sigma_{\beta}^{+}\right)}$is a centrally symmetric set, whose center $\vec{C}$ satisfies $p_{\mathcal{H}_{j}}(\vec{C})=\frac{[\beta]}{2\left(1-\alpha_{j}\right)}$, for all $j \in J$.

Proof: First, note that $\tau\left(\Sigma_{\beta}^{j}\right)$ is a bounded set of $\mathbb{R}^{r} \times \mathbb{C}^{s} \simeq \mathbb{R}^{d-1}$, and that $p_{\mathcal{H}_{j}}\left(\overline{\tau\left(\Sigma_{\beta}^{+}\right)}\right)=\overline{\tau_{j}\left(\Sigma_{\beta}^{+}\right)}$. Let $\vec{X} \in \tau\left(\Sigma_{\beta}^{+}\right)$. There exists a sequence $\left(\varepsilon_{n}\right)_{n \in \mathbb{N}} \in \mathcal{A}_{\beta}^{\mathbb{N}}$ such that $p_{\mathcal{H}_{j}}(\vec{X})=\sum_{n \in \mathbb{N}} \varepsilon_{n} \alpha_{j}^{n}$ for all $j \in J$. Let $\left(\delta_{n}\right)_{n \in \mathbb{N}} \in \mathcal{A}_{\beta}^{\mathbb{N}}$ be defined by $\delta_{n}=[\beta]-\varepsilon_{n}$ for all $n \in \mathbb{N}$. We define $\vec{Y} \in \mathbb{R}^{r} \times \mathbb{C}^{s}$ by $p_{\mathcal{H}_{j}}(\vec{Y})=\sum_{n \in \mathbb{N}} \delta_{n} \alpha_{j}^{n}$ for all $j \in J$. Then $p_{\mathcal{H}_{j}}(\vec{Y})=\frac{[\beta]}{1-\alpha_{j}}-p_{\mathcal{H}_{j}}(\vec{X})$ for all $j \in J$. Moreover, $\vec{Y}$ can be described as the limit of a sequence $\left(\overrightarrow{Y_{n}}\right)_{n \in \mathbb{N}} \in \tau\left(\Sigma_{\beta}^{j}\right)$, whose elements are defined by $p_{\mathcal{H}_{j}}\left(\overrightarrow{Y_{n}}\right)=\sum_{k=0}^{n} \delta_{k} \alpha_{j}^{k}$ for all $j \in J$. Since $\tau\left(\Sigma_{\beta}^{j}\right)$ is a compact set, we deduce that $\vec{Y} \in \mathcal{T}$. Hence, if we define $\vec{C} \in \mathbb{R}^{r} \times \mathbb{C}^{s}$ by $p_{\mathcal{H}_{j}}(\vec{C})=\frac{[\beta]}{2\left(1-\alpha_{j}\right)}$ for all $j \in J$, we obtain that $\overline{\tau\left(\Sigma_{\beta}^{+}\right)}$is stable under the symmetry of center $\vec{C}$, which ends the proof.

Definition 2.4 The symmetrized tile is defined as $\mathcal{T}^{\prime}=\overline{\tau\left(\Sigma_{\beta}^{+}\right)}$.

One directly deduces from Proposition 2.1 and Lemma 2.3 the following:

Proposition 2.5 Let $\beta$ be a confluent Parry number. Then $\mathcal{T}$ is a centrally symmetric set.

Remark 5 When $\beta$ is the sofic Parry number defined by the polynomial $X^{2}-\left(k_{1}+1\right) X+k_{1}-k_{2}$ with $k_{1}>k_{2}>0$, one has $d_{\beta}(1)=0 . k_{1} k_{2}^{\infty}, \mathcal{T}=\left[0, \frac{k_{1} \beta^{2}}{\beta^{2}-k_{2}^{2}}\right]$ and $\mathcal{T}^{\prime}=\left[0, \frac{k_{1} \beta}{\beta-k_{2}}\right]$. Hence $\mathcal{T}$ is a centrally symmetric set with $\mathcal{T} \subsetneq \mathcal{T}^{\prime}$. We do not know whether there exist others Parry numbers for which $\mathcal{T}$ is centrally symmetric with $\mathcal{T} \subsetneq \mathcal{T}^{\prime}$.

The following proposition characterizes centrally symmetrical Rauzy fractals that contain their center $\vec{C}$. Since there exist Rauzy fractals that are not connected, it is not clear whether $\vec{C} \in \mathcal{T}$ for any confluent Parry number.

Proposition 2.6 Let $\beta$ be a confluent Parry number. If either $k_{1}$ or $k_{1}-k_{d}$ is even, then $\vec{C} \in \mathcal{T}$.

Proof: Let $\beta$ be a confluent Parry number. We consider the two cases $k_{1}$ even and $k_{1}$ odd. When $k_{1}$ is even, $\frac{k_{1}}{2} \in \mathcal{A}_{\beta}$ and $\left(\sum_{i=0}^{n} \frac{k_{1}}{2} \beta^{i}\right)_{n \in \mathbb{N}}$ is a sequence of $\beta$-integers. For any $j \in J$, the image under $\tau_{j}$ of the elements of this sequence is $\left(\sum_{i=0}^{n} \frac{k_{1}}{2} \alpha_{j}^{i}\right)_{n \in \mathbb{N}}$, which tends to $\frac{k_{1}}{2} \frac{1}{1-\alpha_{j}}$. Since $k_{1}=[\beta]$, we get $\frac{k_{1}}{2} \frac{1}{1-\alpha_{j}}=p_{\mathcal{H}_{j}}(\vec{C})$ according to Lemma 2.3. Since $\mathcal{T}$ is closed, we deduce that $\vec{C} \in \mathcal{T}$.

Suppose now that $k_{1}$ is odd, with $\beta$ root of $X^{d}-k_{1} \sum_{i=1}^{d-1} X^{i}-k_{d}$. If $k_{1}-k_{d}$ is even, we have $\sum_{i=0}^{d-1} \frac{k_{1}}{2} \beta^{i}=\frac{1}{2} \beta^{d}+\frac{k_{1}-k_{d}}{2}$. One has $\sum_{i \in \mathbb{N}} \frac{k_{1}}{2} \alpha_{j}^{i}=\sum_{l \in \mathbb{N}}\left(\sum_{i=0}^{d} \frac{k_{1}}{2} \alpha_{j}^{i+l(d+1)}\right)=\sum_{l \in \mathbb{N}}\left(\frac{k_{1}+1}{2} \alpha_{j}^{d}+\frac{k_{1}-k_{d}}{2}\right) \alpha_{j}^{l(d+1)}$. Let $\left(\overrightarrow{C_{n}}\right)_{n \in \mathbb{N}} \in\left(\mathbb{R}^{d-1}\right)^{\mathbb{N}}$ be defined by $p_{\mathcal{H}_{j}}\left(\overrightarrow{C_{n}}\right)=\sum_{l=0}^{n}\left(\frac{k_{1}+1}{2} \alpha_{j}^{d}+\frac{k_{1}-k_{d}}{2}\right) \alpha_{j}^{l(d+1)}$ for all $j \in J$ and for all $n \in \mathbb{N}$. Since $k_{1}-k_{d}$ and $k_{1}+1$ are even, with $k_{1} \geq k_{d} \geq 1, \frac{k_{1}+1}{2}$ and $\frac{k_{1}-k_{d}}{2} \in \mathcal{A}_{\beta}$. Additionally, the word $\left(\frac{k_{1}+1}{2} 0^{d-1} \frac{k_{1}-k_{d}}{2}\right)^{n}$ is admissible for all $n \in \mathbb{N}$. This means that the sequence of $\beta$-integers $\left(p_{n}\right)_{n \in \mathbb{N}}$, defined by $p_{n}=\sum_{l=0}^{n}\left(\frac{k_{1}+1}{2} \beta^{d}+\frac{k_{1}-k_{d}}{2}\right) \beta^{l(d+1)}$, satisfies $\tau\left(p_{n}\right)=\overrightarrow{C_{n}}$ for all $n \in \mathbb{N}$, hence $\overrightarrow{C_{n}} \in \mathcal{T}$ for all $n \in \mathbb{N}$. Using again Lemma 2.3 and the compactness of $\mathcal{T}$, we deduce that $\vec{C} \in \mathcal{T}$.

Remark 6 Since $p_{\mathcal{H}_{j}}(\vec{C})=\frac{[\beta]}{2\left(1-\alpha_{j}\right)}$ for any $j \in J, \vec{C} \neq \overrightarrow{0}$ 
By the way, let us give a geometric interpretation of Proposition 2.5. Assume that $\beta$ is a confluent Parry number, with $d_{\beta}(1)=0 . k_{1}^{d-1} k_{d}$. According to Proposition 2.1, the associated substitutive language $\mathcal{L}_{\sigma}$ defined by the substitution $\sigma_{\beta}$ is stable under the mirror image map. Moreover, there exist infinitely many palindromic prefixes for $\omega$, the fixed point of $\sigma_{\beta}$. Indeed, let $\left(\omega_{v_{n}}\right)_{n \in \mathbb{N}}$ be the sequence defined by $\omega_{v_{n}}=h^{n}\left(a_{1}\right)$ for all $n \in \mathbb{N}$, where the map $h$ is defined by

$$
h: \mathcal{L}_{\sigma} \longrightarrow \mathcal{L}_{\sigma}, v \longmapsto \sigma_{\beta}(v) a_{1}^{k_{1}}
$$

One checks that $h$ preserves the set of palindromes of $\mathcal{L}_{\sigma}$, which implies that $\left(\omega_{v_{n}}\right)_{n \in \mathbb{N}}$ is a sequence of palindromic prefixes of $\omega$. Let $v \in \mathcal{A}$ and $x=p_{\mathcal{D}}(f(v))$, where $f$ denotes the abelianization map. We remind that, by definition of the map $p_{\mathcal{D}}$ defined in Section 1.4, one has $p_{\mathcal{D}}\left(f\left(a_{1}\right)\right)=1$ and $p_{\mathcal{D}}(\sigma(w))=\beta \cdot p_{\mathcal{D}}(f(w))$ for all $w \in \mathcal{L}_{\sigma}$. Thus, one has $p_{\mathcal{D}}(f(h(v)))=\beta x+k_{1}$, hence for all $n \in \mathbb{N}, p_{\mathcal{D}}\left(f\left(h^{n}\left(a_{1}\right)\right)\right)=g^{n}(1)$, where $g: \mathbb{R} \rightarrow \mathbb{R}$ is the affine map defined by $g(x)=\beta x+k_{1}$.

Since $\omega_{v_{n}}$ is a palindrome, the set $E_{n}=\left\{f\left(\omega_{k}\right), k \in \llbracket 0, \ldots, v_{n} \rrbracket\right\}$ is a centrally symmetrical set of $\mathbb{Z}^{d}$. Hence $p_{\mathcal{D}}\left(E_{n}\right)$ and $p_{\mathcal{H}}\left(E_{n}\right)$ respectively are centrally symmetrical sets of $\mathbb{R}$ and $\mathbb{R}^{d-1}$. Let $\overrightarrow{C_{n}}$ denote the center of $p_{\mathcal{H}}\left(E_{n}\right)$. Then for all $j \in J, p_{\mathcal{H}_{j}}\left(\overrightarrow{C_{n}}\right)=\frac{g_{j}^{v_{n}}(1)}{2}$, where $g_{j}$ is the affine map: $x \longmapsto \alpha_{j} x+k_{1}$. Let $c_{j}$ be the fixed point of $g_{j}$ for all $j \in J$. Since $\beta$ is a Pisot number, $g_{j}$ is a contracting map for all $j \in J$, hence the sequence $\left(p_{\mathcal{H}_{j}}\left(\overrightarrow{C_{n}}\right)\right)_{n \in \mathbb{N}}$ tends to $\frac{c_{j}}{2}$. Let $\vec{C}$ be the limit of the sequence $\left(\overrightarrow{C_{n}}\right)_{n \in \mathbb{N}}$. Since $p_{\mathcal{H}_{j}}(\vec{C})=\frac{c_{j}}{2}$, and since $k_{1}=[\beta]$, the center of $\mathcal{T}$ satisfies $p_{\mathcal{H}_{j}}(\vec{C})=\frac{[\beta]}{2\left(1-\alpha_{j}\right)}$ for all $j \in J$. We retrieve the result of Lemma 2.3 , since $\mathcal{T}=\mathcal{T}^{\prime}$. Note that, rather than the foregoing construction of $\mathcal{T}$ as the limit of centrally symmetric sets, constructed by considering prefixes of the fixed point $\omega$ of $\sigma_{\beta}$, it is also possible to describe $\mathcal{T}$ as the image of the closure of $p_{\mathcal{H}}\left(f\left(\tilde{\omega}^{\prime} \cdot \omega^{\prime}\right)\right)$ under a translation map, where $\tilde{\omega}^{\prime} \cdot \omega^{\prime}$ is a two-sided palindrome whose factors belong to $\mathcal{L}_{\sigma}$. This construction, and the previous one, cannot be completed when $\beta$ is a Parry number which is not confluent, since $\mathcal{L}_{\sigma}$ contains only finitely many palindromes in this case.

There is no reason for the tile $\mathcal{T}$ to be a centrally symmetric set when $\beta$ is a Parry number which is not confluent. See for instance Figure 2 and Figure 3, which respectively represent, for the numeration system defined by $d_{\beta}(1)=0.101$, first, the Rauzy fractal $\mathcal{T}$, then the sets $\mathcal{T} \cup S_{\vec{C}}(\mathcal{T})$ and $\mathcal{T}^{\prime}$ with different levels of shading.

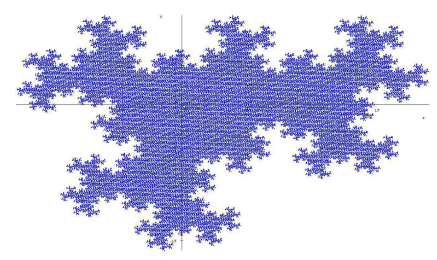

Fig. 2: $\mathcal{T}$ defined by $d_{\beta}(1)=0.101$

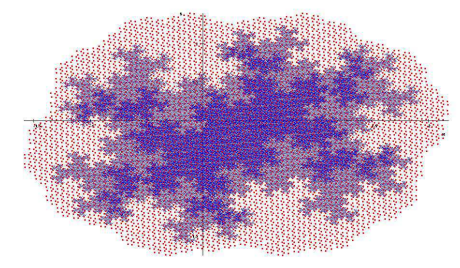

Fig. 3: $\mathcal{T}^{\prime}$ defined by $d_{\beta}(1)=0.101$

\section{Computation of $L_{\oplus}$ in several cases of cubic Pisot numbers}

In this section, we are interested in computing $L_{\oplus}$, or at least computing a better upper bound for $L_{\oplus}$ than those determined in [9]. First, we focus in 3.1 on several algebraic inequalities, provided by Propositions 3.4 and 3.5, that are satisfied by the image under $\tau$ of the finite $\beta$-fractional parts that are obtained as the $\beta$-fractional parts of the sum of two $\beta$-integers. Whereas these relations do not allow us to compute directly the set of these finite $\beta$-fractional parts, we can discard, thanks to an algorithm defined in Section 3.2, the $\beta$-fractional parts which do not satisfy these inequalities. In particular, this allows us to compute $L_{\oplus}$ for several cases of Pisot units, since, among the remaining $\beta$-fractional parts that were not discarded by the algorithm $A$, we find an element of $\operatorname{Fin}(\beta)$, whose $\beta$-fractional part is of length $L_{\oplus}$, which is the sum of two $\beta$-integers. 


\subsection{Fractional parts of sums or differences of $\beta$-integers}

Let $\beta$ be a Pisot number, and let $\left(w_{i}\right)_{i \in \mathbb{N}} \in \mathcal{A}_{\beta}^{\mathbb{N}}$. Let $\left(v_{i}\right)_{i \in \mathbb{N}}$ be defined for all $i \in \mathbb{N}$ by $v_{i}=[\beta]-w_{i}$. Since $\left(v_{i}\right)_{i \in \mathbb{N}} \in \mathcal{A}_{\beta}^{\mathbb{N}}, \mathcal{T}^{\prime}=\overline{\tau\left(\Sigma_{\beta}^{+}\right)}$contains both $\mathcal{T}$ and $S_{\vec{C}}(\mathcal{T})=\bigcup_{\vec{X} \in \mathcal{T}}\{2 \vec{C}-\vec{X}\}$, where $\vec{C}$ is the center of $\mathcal{T}^{\prime}$. We deduce from $\mathcal{T} \subset \mathcal{T}^{\prime}$ that inequalities satisfied by elements of $\mathcal{T}^{\prime}$ are satisfied by elements of $\mathcal{T}$ as well. Note that there is no reason that $\mathcal{T} \cup S_{\vec{C}}(\mathcal{T})=\mathcal{T}^{\prime}$, see Figure 3 .

First, let us recall some results of [9], where a method is described in order to compute upper bounds for $L_{\oplus}$ and $L_{\odot}$. Let $\alpha_{j}$ denote a Galois conjugate of $\beta$ such that $\left|\alpha_{j}\right|<1$. We define for all $n \in \mathbb{N}^{*}$ the real numbers

$$
M_{n}(j)=\max _{x \in \mathbb{Z}_{\beta}^{+}}\left\{\left|\tau_{j}(x)\right|, x<\beta^{n}\right\}, H_{n}(j)=\frac{M_{n}(j)}{1-\left|\alpha_{j}\right|^{n}} \text { and } H(j)=\sup _{x \in \mathbb{Z}_{\beta}^{+}}\left|\tau_{j}(x)\right| .
$$

Let us give a geometric interpretation for $H_{n}(j)$. Indeed, $H_{n}(j)$ is the upper bound of the modulus of complex numbers $z \in \mathcal{T}$ such that, for all $j \in J, p_{\mathcal{H}_{j}}(z)$ admits a periodic $\alpha_{j}$-expansion of period $n$. Hence, for all $j \in J, H_{l}(j) \geq H_{k}(j)$ for all $k, l \in \mathbb{N}$ such that $l \mid k$, and $H_{k}(j) \geq H(j)$ for all $k \in \mathbb{N}^{*}$.

The following inequality, used for instance in the proof of Proposition 3.2, holds.

Proposition 3.1 For all $x \in \mathbb{Z}_{\beta}$ and $n \in \mathbb{N}^{*},\left|\tau_{j}(x)\right|<H_{n}(j)$.

Proposition 3.1 allows us to obtain the following result, which provides upper bounds for $L_{\oplus}$ and $L_{\odot}$.

Proposition $3.2[9]$ Let $\beta>1$ be an algebraic integer. If there exists a Galois conjugate $\alpha_{j}$ of $\beta$ such that $K(j)=\inf _{z \in \mathbb{Z}_{\beta}^{+} \backslash \beta \mathbb{Z}_{\beta}^{+}}\left|\tau_{j}(z)\right|>0$, then

$$
L_{\oplus}<\frac{\ln 2+\ln H(j)-\ln K(j)}{-\ln \left|\alpha_{j}\right|} \text { and } L_{\odot}<\frac{2 \ln H(j)-\ln K(j)}{-\ln \left|\alpha_{j}\right|} .
$$

In particular, assume that $d_{\beta}(1)=0 . k_{1} k_{2} k_{3}$, and that $\beta$ has two complex Galois conjugates. Then $\beta$ is a Pisot cubic number; the finiteness property holds [3], which implies due to [2] that 0 is an inner point of $\mathcal{T}$. Hence $K(j)>0$, and the required conditions of Proposition 3.2 are satisfied.

Proposition 3.2 is a consequence of Proposition 3.1. Indeed, let $x$ and $y \in \mathbb{Z}_{\beta}$ with $x+y \in \operatorname{Fin}(\beta)$. Since $l_{f}(x+y) \leq L_{\oplus}$, one gets $\left|\tau_{j}(x)\right|+\left|\tau_{j}(y)\right|<2 H(j)$ and $\left|\tau_{j}(x+y)\right|>\left|\alpha_{j}\right|^{L_{\oplus}} K(j)$. Hence the upper bound for $L_{\oplus}$, and the case of multiplication is performed in a similar way. We focus now on the search of better inequalities satisfied by elements of $\operatorname{Fin}(\beta)$, since this will allow us to deduce better upper bounds for $L_{\oplus}$ and $L_{\odot}$. Inequalities improving Proposition 3.1 are detailed below in Propositions 3.4 and 3.5.

Since $\mathbb{Z}_{\beta}^{+} \subset \Sigma_{\beta}^{+}$, any inequality satisfied by elements of $\tau\left(\Sigma_{\beta}^{+}\right)$is satisfied by $\tau\left(\mathbb{Z}_{\beta}^{+}\right)$as well. Hence the study of $\tau\left(\Sigma_{\beta}^{+}\right)$will provide additional inequalities satisfied by elements of $\mathcal{T}$. First, we define for all $n \in \mathbb{N}^{*}$ the real numbers

$$
M_{n}^{\prime}(j)=\max _{x \in \Sigma_{\beta}^{+}}\left\{\left|\tau_{j}(x)\right|, x<\beta^{n}\right\}, H_{n}^{\prime}(j)=\frac{M_{n}^{\prime}(j)}{1-\left|\alpha_{j}\right|^{n}} \text { and } H^{\prime}(j)=\sup _{x \in \Sigma_{\beta}^{+}}\left|\tau_{j}(x)\right| .
$$

Similarly as in [9], we obtain using the triangle inequality the following property.

Lemma 3.3 For all $x \in \mathbb{Z}_{\beta},\left|\tau_{j}(x)\right|<H_{n}^{\prime}(j)$ and $\left|\tau_{j}(x)-2 p_{\mathcal{H}_{j}}(\vec{C})\right|<H_{n}^{\prime}(j)$.

Proof: The first assertion is deduced from Proposition 3.1, whereas the second assertion is a consequence of $S_{\vec{C}}(\mathcal{T}) \subset \mathcal{T}^{\prime}$. 
Note that, since $H^{\prime}(j) \geq H(j)$, the first inequality of Lemma 3.3 is a weaker condition than the inequality of Proposition 3.1. However, it is more convenient to compute $H^{\prime}(j)$, since the definitions of $H_{n}^{\prime}(j)$ and $H^{\prime}(j)$ do not use the admissibility condition on words.

We obtain additional algebraic inequalities, satisfied by finite $\beta$-fractional parts of the sums of two $\beta$-integers, thanks to the following proposition.

Proposition 3.4 Let $x, y \in \mathbb{Z}_{\beta}^{+}$with $x \geq y$. If $x+y \in \operatorname{Fin}(\beta)$, then:

$$
\begin{aligned}
& \text { 1. }\left|\tau_{j}\left(\{x+y\}_{\beta}\right)-2 p_{\mathcal{H}_{j}}(\vec{C})\right|<3 H^{\prime}(j) ; \\
& \text { 2. }\left|\tau_{j}\left(\{x+y\}_{\beta}\right)\right|<\left(2+\left|\alpha_{j}\right|\right) H(j) . \\
& \text { If } x-y \in \operatorname{Fin}(\beta) \text {, then: } \\
& \text { 1. }\left|\tau_{j}\left(\{x-y\}_{\beta}\right)+2 p_{\mathcal{H}_{j}}(\vec{C})\right|<3 H^{\prime}(j) \text {; } \\
& \text { 2. }\left|\tau_{j}\left(\{x-y\}_{\beta}\right)\right|<\left(2+\left|\alpha_{j}\right|\right) H(j) .
\end{aligned}
$$

Proof: First, let us consider the case of addition of positive $\beta$-integers. Let $x, y \in \mathbb{Z}_{\beta}^{+}$such that $x+y \in \operatorname{Fin}(\beta)$. The first assertion is a consequence of Lemma 3.3, using the fact that, since $x$, $y$ and $[x+y]_{\beta}$ are $\beta$-integers, we get $\left|\tau_{j}(x)-2 p_{\mathcal{H}_{j}}(\vec{C})\right|<H^{\prime}(j),\left|\tau_{j}(y)-2 p_{\mathcal{H}_{j}}(\vec{C})\right|<H^{\prime}(j)$ and $\left|\tau_{j}\left([x+y]_{\beta}\right)-2 p_{\mathcal{H}_{j}}(\vec{C})\right|<H^{\prime}(j)$. Let us prove the second assertion.

If 0 is a suffix of the expansion of either $x, y$, or $[x+y]_{\beta}$, then one of these three $\beta$-integers, say $x$, satisfies $\left|\tau_{j}(x)\right|<\left|\alpha_{j}\right| H(j)$, which improves the inequality of the first assertion. On the other hand, suppose that none of the $\beta$-expansions of $x, y$ and $[x+y]_{\beta}$ admits 0 as a suffix. Denote respectively by $x_{0}, y_{0}$ and $z_{0}$ the suffixes of $x, y$ and $[x+y]_{\beta}$. Then, one of the following nonnegative $\beta$-integers $x^{\prime}=x-\min \left\{x_{0}, y_{0}\right\}, y^{\prime}=y-\min \left\{x_{0}, y_{0}\right\}$ or $z^{\prime}=y-\min \left\{x_{0}, y_{0}\right\}$ admits 0 as a suffix, and both $x^{\prime}+y=z^{\prime}+\{x+y\}_{\beta}$ and $x+y^{\prime}=z^{\prime}+\{x+y\}_{\beta}$ hold. Among the $\beta$-integers $x, x^{\prime}, y, y^{\prime}$, one of them belongs to $\beta \mathbb{Z}_{\beta}^{+}$. This proves that there exist $x^{\prime}, y^{\prime} \in \mathbb{Z}_{\beta}^{+}$, with either $x^{\prime}$ or $y^{\prime} \in \beta \mathbb{Z}_{\beta}^{+}$, such that $\{x+y\}_{\beta}=\left\{x^{\prime}+y^{\prime}\right\}_{\beta}$, hence $\left|\tau_{j}\left(\{x+y\}_{\beta}\right)\right|<\left(2+\left|\alpha_{j}\right|\right) H(j)$.

The case of the subtraction of two positive $\beta$-integers is handled in the same way.

These relations improve those found in [9]. We obtain Proposition 3.5 in a similar way, which enables the computation of $L_{\oplus}$ in Section 3.2 for several cases of cubic Pisot units.

Remark 7 Proposition 3.4 means that $3 H^{\prime}(j)$ and $\left(2+\left|\alpha_{j}\right|\right) H(j)$ are both upper bounds for $\left|\tau_{j}\left(\{x+y\}_{\beta}\right)\right|$ when $x$ and $y$ are $\beta$-integers such that $[x+y]_{\beta} \in$ Fin $(\beta)$. When $\beta$ is a confluent Parry number, $H(j)=H^{\prime}(j)$, which means that $\left(2+\left|\alpha_{j}\right|\right) H(j)$ provides a better upper bound for $\left|\tau_{j}\left(\{x+y\}_{\beta}\right)\right|$ than $3 H^{\prime}(j)$. However, when $\beta$ is the positive root of the polynomial $X^{3}-X^{2}-1$, we check by pure computation that $3 H^{\prime}(j)<\left(2+\left|\alpha_{j}\right|\right) H(j)$. Hence, in the general case of Pisot numbers, we do not know which of the two upper bounds $3 H^{\prime}(j)$ and $\left(2+\left|\alpha_{j}\right|\right) H(j)$ for $\left|\tau_{j}\left(\{x+y\}_{\beta}\right)\right|$ is better than the other one.

The following proposition is an improvement of Proposition 3.4, in the sense that it sums up the inequalities concerning the elements of $\mathcal{T}$. In particular, we obtain as a result an accurate covering of $\mathcal{T}$ by balls having arbitrarily small positive radius. As a consequence, we construct the algorithm $A$ described in Section 3.2, which computes a set of admissible words whose $\beta$-fractional parts are of a bounded length. This set contains the finite $\beta$-fractional parts of sums of two positive $\beta$-integers.

Proposition 3.5 Let $x, y \in \mathbb{Z}_{\beta}^{+}$with $x>y, x=\sum_{k \in \mathbb{N}} \varepsilon_{k} \beta^{k}$ and $y=\sum_{k \in \mathbb{N}} \delta_{k} \beta^{k}$. Suppose that $x+y \in \operatorname{Fin}(\beta)$. Let $\left(\nu_{k}\right)_{k \in \mathbb{Z}}$ be the $\beta$-expansion of $x+y$. Then, for all $N \in \mathbb{N}^{*}$ :

$$
\left|\sum_{k<0} \nu_{k} \alpha_{j}^{k}+\sum_{k=0}^{N-1}\left(\nu_{k}-\varepsilon_{k}-\delta_{k}\right) \alpha_{j}^{k}-\alpha_{j}^{N} p_{\mathcal{H}_{j}}(\vec{C})\right|<\frac{3 H^{\prime}(j)\left(1+\left|\alpha_{j}\right|\right)}{2}\left|\alpha_{j}\right|^{N} .
$$


Proof: First, we know due to Lemma 2.3 that $\vec{C}$ is the center of the centrally symmetric set $\mathcal{T}^{\prime}$, with $p_{\mathcal{H}_{j}}(\vec{C})=\frac{[\beta]}{2\left(1-\alpha_{j}\right)}=\sum_{k \geq 0} \frac{[\beta]}{2} \alpha_{j}^{k}$ for all $j \in J$.

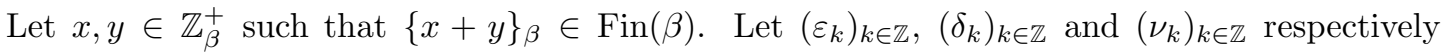
be the $\beta$-expansion of $x, y$ and $x+y$. We remind that, by hypothesis, $\varepsilon_{k}=\delta_{k}=0$ for all $k<0$, and that $\left(\varepsilon_{k}-\delta_{k}-\nu_{k}\right)_{k \in \mathbb{Z}}$ consists of only finitely many non-zeros elements. Since $\{x+y\}_{\beta}+[x+y]_{\beta}-x-y=0$, one gets for all $N \in \mathbb{N}^{*}$ :

$$
\begin{aligned}
\sum_{k<0} \nu_{k} \alpha_{j}^{k}+\sum_{k=0}^{N-1}\left(\nu_{k}-\varepsilon_{k}-\delta_{k}\right) \alpha_{j}^{k} & =-\sum_{k \geq N}\left(\nu_{k}-\varepsilon_{k}-\delta_{k}\right) \alpha_{j}^{k} \\
& =-\alpha_{j}^{N} \sum_{k \in \mathbb{N}}\left(\nu_{k+N}-\varepsilon_{k+N}-\delta_{k+N}\right) \alpha_{j}^{k}
\end{aligned}
$$

Since for all $j \in J$, one has $p_{\mathcal{H}_{j}}(\vec{C})=\sum_{k \in \mathbb{N}} \frac{[\beta]}{2} \alpha_{j}^{k}=\frac{[\beta]}{2\left(1-\alpha_{j}\right)}$, we obtain:

$$
\sum_{k \geq N}\left(\nu_{k}-\varepsilon_{k}-\delta_{k}\right) \alpha_{j}^{k}=\alpha_{j}^{N}\left(\sum_{k \in \mathbb{N}}\left(\nu_{k+N}-\varepsilon_{k+N}-\delta_{k+N}+\frac{[\beta]}{2}\right) \alpha_{j}^{k}-p_{\mathcal{H}_{j}}(\vec{C})\right)
$$

Moreover, $\nu_{k+N}-\varepsilon_{k+N}-\delta_{k+N}+\frac{[\beta]}{2} \in\left[-3 \frac{[\beta]}{2}, 3 \frac{[\beta]}{2}\right]$ for all $k \in \mathbb{N}$. Hence

$$
\left|\sum_{k<0} \nu_{k} \alpha_{j}^{k}+\sum_{k=0}^{N-1}\left(\nu_{k}-\varepsilon_{k}-\delta_{k}\right) \alpha_{j}^{k}-\alpha_{j}^{N} p_{\mathcal{H}}(\vec{C})\right|<\frac{3}{2}\left|\alpha_{j}\right|^{N} R
$$

where $R=\sup \left\{\left|\sum_{k \in \mathbb{N}} v_{k} \alpha_{j}^{k}\right|,\left(v_{k}\right)_{k \in \mathbb{N}} \in[-[\beta],[\beta]]^{\mathbb{N}}\right\}$.

Since $\overline{\left\{\sum_{k \in \mathbb{N}} v_{k} \alpha_{j}^{k},\left(v_{k}\right)_{k \in \mathbb{N}} \in[-[\beta],[\beta]]^{\mathbb{N}}\right\}}$ is a convex set, and using a similar argument as in the proof of Proposition 3.4, we get:

$$
R=\max _{x^{\prime}, y^{\prime} \in \Sigma_{\beta}^{+}}\left|\tau_{j}\left(x^{\prime}-y^{\prime}\right)\right| \leq\left(1+\left|\alpha_{j}\right|\right) H^{\prime}(j) .
$$

Note that we do not use the admissibility in the proof of Propositions 3.4 and 3.5. This means that, whereas we are looking for inequalities concerning elements which belong to $\mathcal{T}$, these inequalities hold in fact for elements of $\mathcal{T}^{\prime}$.

The relation given by Proposition 3.5 produces a good approximation of $\mathcal{T}^{\prime}$, in the sense that it enables an accurate covering of $\mathcal{T}^{\prime}$ with balls having arbitrarily small positive radius. More precisely, the coordinates on $\mathcal{H}_{j}$ of the centers of these balls are $\sum_{k=0}^{N-1}\left(\nu_{k}-\varepsilon_{k}-\delta_{k}\right) \alpha_{j}^{k}-p_{\mathcal{H}_{j}}(\vec{C})$ when $\nu, \varepsilon$ and $\delta$ describe the set of admissible words of length $N$, and their radii are $\frac{3}{2}\left|\alpha_{j}\right|^{N} R$.

It could be possible that the set $\mathcal{T}$ is only a "small" subset of $\mathcal{T}$ '. In this case, the inequality satisfied by elements of $\mathcal{T}$ in Proposition 3.5 is not accurate enough, and we need to improve the relation satisfied by elements of $\mathcal{T}$ in order to compute the exact value of $L_{\oplus}$, or at least a better upper bound for $L_{\oplus}$ than those given by Proposition 3.2. Note that the class of confluent Parry numbers is in some sense optimal, since $\mathcal{T}=\mathcal{T}^{\prime}$ corresponds to the case where a good approximation of $\mathcal{T}^{\prime}$ is a good approximation of $\mathcal{T}$ as well.

\subsection{Algorithmic computation of $L_{\oplus}$ in several cubic Pisot cases}

In this section, we are interested in the set of finite $\beta$-fractional parts that are obtained when one sums two positive $\beta$-integers. We introduce $L_{\oplus}^{+}$as the maximal length of the finite $\beta$-fractional parts of the sum of two positive $\beta$-integers. Note that $L_{\oplus}$, the maximal length of the finite $\beta$ fractional parts of the sum of two $\beta$-integers, can also be defined as the maximal length of the finite 
$\beta$-fractional parts that we obtain after adding or subtracting two positive $\beta$-integers. Hence, if one can both compute $L_{\oplus}^{+}$and the maximal length of the finite $\beta$-fractional parts of the difference of two positive $\beta$-integers, we deduce the value of $L_{\oplus}$.

Let $\mathcal{L}_{\beta}^{\prime}$ be the set which consists of words $\left(\nu_{k}-\varepsilon_{k}-\delta_{k}\right)_{k \in \llbracket 1, \ldots, n \rrbracket}$, where the words $\left(\nu_{k}\right)_{k \in \llbracket 1, \ldots, n \rrbracket}$, $\left(\varepsilon_{k}\right)_{k \in \llbracket 1, \ldots, n \rrbracket}$ and $\left(\delta_{k}\right)_{k \in \llbracket 1, \ldots, n \rrbracket}$ belong to $\mathcal{L}_{\beta}$. Note that the words of $\mathcal{L}_{\beta}^{\prime}$ are defined on the alphabet $\llbracket-2[\beta], \ldots,[\beta] \rrbracket$. Let $x, y \in \mathbb{Z}_{\beta}^{+}$. Then the expansion in base $\beta$ of $x+y-[x+y]_{\beta}$ constructed digit by digit belongs to $\mathcal{L}_{\beta}^{\prime}$, and it represents an element of $[0,1[$.

In order to obtain a better upper bound for $L_{\oplus}$ than those computed in [9], we define the following algorithm $A$.

1. We set for $B$ an upper bound for $L_{\oplus}$, for instance the upper bound computed in Proposition 3.2 , and we set $N=1$.

2. We check whether there exists words $v=v_{B} \ldots v_{1} \in \mathcal{L}_{\beta}$ and $w=w_{0} \ldots w_{N-1}$ in $\mathcal{L}_{\beta}^{\prime}$ such that the inequality given by Proposition 3.5 holds, that is, if we have for all $j \in J$

$$
\left|\sum_{k=1}^{B} v_{k} \alpha_{j}^{-k}+\sum_{k=0}^{N-1} w_{k} \alpha_{j}^{k}-\alpha_{j}^{N} p_{j}(\vec{C})\right|<\frac{3 H^{\prime}(j)\left(1+\left|\alpha_{j}\right|\right)}{2}|\alpha|^{N} .
$$

3. When (3) is satisfied for no $v \in \mathcal{L}_{\beta}$ and no $w \in \mathcal{L}_{\beta}^{\prime}$, we decrease by 1 the value of $B$, we set $N=1$ and we return to point 2 .

4. When there exists $v \in \mathcal{L}_{\beta}$ and $w \in \mathcal{L}_{\beta}^{\prime}$ such that (3) holds, we increase by 1 the value of $N$ and we return to point 2 .

Since $B \in \mathbb{N}$, there eventually exists an admissible word $v$ such that (3) is satisfied for any $w \in \mathcal{L}_{\beta}^{\prime}$. This algorithm lowers the value of $B$, that is, it provides a better upper bound for $L_{\oplus}^{+}$. However, we cannot check if this algorithm computes the value of $L_{\oplus}^{+}$. Additionally, even if the algorithm computes the exact value of $L_{\oplus}^{+}$after finitely many steps, we do not known when the exact value of $L_{\oplus}^{+}$is reached. In this case, it suffices then to find two $\beta$-integers $x$ and $y$ such that $d_{\beta}(x+y)$ has a fractional part of the required length to deduce the exact value of $L_{\oplus}^{+}$. We finally perform the same computations for the case of the difference of two positive $\beta$-integers in order to obtain $L_{\oplus}$.

Let us focus now on the case of cubic Pisot numbers. Let $\beta$ be the positive root of the polynomial $P(X)=X^{3}-k_{1} X^{2}-k_{2} X-1$, where $k_{1}, k_{2} \in \mathbb{N}^{2}$ satisfy $k_{1} \geq \max \left\{1, k_{2}\right\}$. Suppose moreover that $P$ has two complex conjugate roots, that we denote by $\alpha$ and $\bar{\alpha}$. For $k_{2}$ large enough, this condition is satisfied when $k_{2} \leq 2 \sqrt{k_{1}}$, and is not satisfied otherwise.

Due to [3], we know that the finiteness property $(\mathcal{F})$ holds in this case. It is also proved in this article that, when $\beta$ is a Pisot unit which satisfies $(\mathcal{F})$, then 0 is an inner point of $\mathcal{T}$. Hence $K=\min _{z \in \mathbb{Z}_{\beta}^{+} \backslash \beta \mathbb{Z}_{\beta}^{+}}|\tau(z)|>0$, and the required conditions of Proposition 3.2 hold for the class of Parry numbers we consider. We respectively denote by $L_{\oplus}\left(k_{1}, k_{2}\right)$ and $L_{\oplus}^{+}\left(k_{1}, k_{2}\right)$ the value of $L_{\oplus}$ and $L_{\oplus}^{+}$for the associated numeration system, defined by $d_{\beta}(1)=0 . k_{1} k_{2} 1$.

Proposition 3.6 The algorithm A computes $L_{\oplus}\left(k_{1}, k_{2}\right)$ when $k_{1} \leq 3$. We get:

1. $L_{\oplus}(1,0)=11$ and $L_{\oplus}^{+}(1,0)=10$,

2. $L_{\oplus}(1,1) \leq 6$,

3. $L_{\oplus}(2,0)=L_{\oplus}^{+}(2,0)=7$,

4. $L_{\oplus}(2,1)=5$ and $L_{\oplus}^{+}(2,1)=4$,

5. $L_{\oplus}(2,2)=L_{\oplus}^{+}(2,2)=5$. 
6. $L_{\oplus}(3,0)=L_{\oplus}^{+}(3,0)=4$,

7. $L_{\oplus}(3,1)=L_{\oplus}^{+}(3,1)=4$,

8. $L_{\oplus}(3,2)=4$ and $L_{\oplus}^{+}(3,2)=3$,

9. $L_{\oplus}(3,3)=L_{\oplus}^{+}(3,3)=5$.

Note that the inequality $L_{\oplus}(1,1) \leq 6$ was already known, see [9].

Example 2 When $d_{\beta}(1)=0.101$, one has $\beta^{13}+\beta^{9}+\beta^{6}+\beta^{3}+1-\left(\beta^{11}+\beta^{8}+\beta^{4}+\beta\right)=$ $\beta^{11}+\beta^{8}+\beta^{4}+\beta+\beta^{-3}+\beta^{-6}+\beta^{-11}$ and $\left(\beta^{4}+1\right)+\left(\beta^{3}+1\right)=\beta^{5}+\beta^{2}+\beta^{-1}+\beta^{-5}+\beta^{-10}$. When $d_{\beta}(1)=0.201$, one has $\left(\beta^{5}+\beta^{4}+\beta+2\right)+\left(\beta^{5}+\beta+2\right)=\beta^{6}+\beta^{3}+2 \beta^{2}+\beta^{-1}+2 \beta^{-2}+\beta^{-5}+\beta^{-7}$.

From a practical point of view, we noticed that, except in the Tribonacci case, the algorithm $A$ computes $L_{\oplus}\left(k_{1}, k_{2}\right)$ in a finite number of steps for any example of a pair $\left(k_{1}, k_{2}\right)$ among those that satisfy the needed conditions, that is, for which $\beta$ is the positive root of the polynomial $X^{3}-k_{1} X^{2}-k_{2} X-1$ and admits two complex Galois conjugates.

\subsection{The particular case of Tribonacci}

We end our study by the Tribonacci case, that is, $\beta$ is the real root of the polynomial $X^{3}-X^{2}-$ $X-1$. Since $\beta$ admits two complex Galois conjugates, we set from now on $\alpha=\alpha_{1}$ and $\tau=\tau_{1}$. Note that this definition of $\tau$ coincides with the previous definition of $\tau$ given in Section 1.4. The previous method does not allow us to determine the exact value of $L_{\oplus}(1,1)$, since the admissible word 100011, which represents the $\beta$-fractional part $\beta^{-1}+\beta^{-5}+\beta^{-6}$ is not discarded by the algorithm described in Section 3.2. Hence we do not know whether there exist two $\beta$-integers $x$ and $y$ such that $\{x+y\}_{\beta}=\beta^{-1}+\beta^{-5}+\beta^{-6}$. This explains why the particular case of Tribonacci needs additional computations.

We prove in this section that $L_{\oplus}(1,1)=5$, thanks to the following lemma.

Lemma 3.7 Let $\beta$ be the Tribonacci number, that is, $d_{\beta}(1)=0.111$. Suppose that there exist $x, y \in \mathbb{Z}_{\beta}^{+}$such that $l_{f}(x+y)=6$. Then, we have:

1. $\{x+y\}_{\beta}=\beta^{-1}+\beta^{-5}+\beta^{-6}$,

2. the real numbers $\frac{x-1}{\beta^{3}}, \frac{y-1}{\beta^{3}}$ and $\frac{[x+y]_{\beta}-\beta^{2}-\beta}{\beta^{3}}$ are $\beta$-integers.

Proof: The first assertion is a consequence of Proposition 3.6, since we prove by pure computation that the relation (3) only holds for $v$ an admissible word of length 6 , namely when $v=100011$. Additionally, we obtain only one possibility for the suffix of length 3 of $w \in \mathcal{L}_{\beta}^{\prime}$, which is $(-1)(-1) 2$. This suffix corresponds to the suffix of length 3 of the expansion in base $\beta$ of $x+y-[x+y]_{\beta}$ constructed digit by digit. This implies that the $\beta$-expansions of the $\beta$-integers $x, y$ and $[x+y]_{\beta}$ must respectively admit 001, 001 and 110 as suffixes, which proves the second assertion.

Proposition 3.8 When $d_{\beta}(1)=0.111$, then $L_{\oplus}^{+}=5$.

Proof: Suppose that there exist $x_{0}$ and $y_{0} \in \mathbb{Z}_{\beta}^{+}$such that $\left\{x_{0}+y_{0}\right\}_{\beta}=\beta^{-1}+\beta^{-5}+\beta^{-6}$. This means that the set $\mathcal{S}_{6}=\left\{(x, y, z) \in\left(\mathbb{Z}_{\beta}^{+}\right)^{3}, x+y=z+\beta^{-1}+\beta^{-5}+\beta^{-6}\right\}$ is not empty. Let $z_{0}=\left[x_{0}+y_{0}\right]_{\beta}$. Let $\left(x_{0}, y_{0}, z_{0}\right) \in \mathcal{S}_{6}$ such that $l_{i}\left(z_{0}\right)$, the length of the $\beta$-fractional part of $z_{0}$, is minimal. Due to Lemma 3.7, there exists $\left(x_{1}, y_{1}, z_{1}\right) \in\left(\mathbb{Z}_{\beta}^{+}\right)^{3}$ such that $x_{0}=1+\beta^{3} x_{1}$, $y_{0}=1+\beta^{3} y_{1}$ and $z_{0}=\beta+\beta^{2}+\beta^{3} z_{1}$. Thus, we get:

$$
\begin{aligned}
\beta^{3}\left(x_{1}+y_{1}\right) & =x_{0}+y_{0}-2 \\
& =z_{0}+\beta^{-1}+\beta^{-5}+\beta^{-6}-2 \\
& =\beta^{3} z_{1}+\beta^{-1}+\beta^{-5}+\beta^{-6}+\beta+\beta^{2}-2 \\
& =\beta^{3} z_{1}+\beta^{2}+\beta^{-2}+\beta^{-3}
\end{aligned}
$$


We deduce that $x_{1}+y_{1}=z_{1}+\beta^{-1}+\beta^{-5}+\beta^{-6}$, hence the 3 -uple $\left(x_{1}, y_{1}, z_{1}\right)$ belongs to $\mathcal{S}_{6}$. However, $l_{i}\left(z_{1}\right) \leq l_{i}\left(z_{0}\right)-3$, which contradicts the minimality of $l_{i}\left(z_{0}\right)$. Hence $\mathcal{S}_{6}$ is empty, and $L_{\oplus}^{+}<6$.

Theorem 3.9 When $d_{\beta}(1)=0.111$, then $L_{\oplus}=5$.

Proof: Proposition 3.8 proves that $L_{\oplus}^{+}=5$. In order to obtain the result on $\mathbb{Z}_{\beta}$ instead of $\mathbb{Z}_{\beta}^{+}$, it suffices to note that, since $\mathbb{Z}_{\beta}= \pm \mathbb{Z}_{\beta}^{+}$, we can suppose that $x+y>0$, thus $[x+y]_{\beta} \in \mathbb{Z}_{\beta}^{+}$without loss of generality. Moreover, if $x+y>0$, then $x$ or $y$ is positive, and the case where they are both positive is exactly the case previously studied. Thus, we only have to check whether there exist $x,-y \in \mathbb{Z}_{\beta}^{+}, x-y>0$ such that $l_{f}(x-y)=6$. However, we check by an exhaustive computation that there exists $N \in \mathbb{N}$ for which the inequality (3) used in the algorithm $A$ does not hold for any admissible word $v$ of length 6 . Since $L_{\oplus} \geq 5$, this proves that $L_{\oplus}=L_{\oplus}^{+}=5$.

Remark 8 Let us explain why the algorithm A described in Section 3.2 does not improve the relation $5 \leq L_{\oplus} \leq 6$ given by [9] in the Tribonacci case. This is due to the fact that the inequality provided by Proposition 3.5, a priori satisfied by images under $\tau_{j}$ of $\beta$-integers, also extends to elements of $\mathcal{T}$. For instance, the complex number $\frac{1}{1-\alpha^{3}}$ belong to $\mathcal{T}$, since it is the limit of the sequence $\left(\sum_{k=0}^{n} \alpha^{3 k}\right)_{n \in \mathbb{N}}$, which consists of images of $\beta$-integers under $\tau$. Hence $\frac{1}{1-\alpha^{3}}+\frac{1}{1-\alpha^{3}}=$ $\frac{2}{1-\alpha^{3}}$ is the sum of two elements which belong to $\mathcal{T}$. Moreover, $\frac{2}{1-\alpha^{3}}$ is the limit of the sequence $\left(\left(\sum_{k=0}^{n} \alpha^{3 k+1}+\alpha^{3 k+2}\right)+\alpha^{-1}+\alpha^{-5}+\alpha^{-6}\right)_{n \in \mathbb{N}}$, which consists of images of elements of Fin $(\beta)$, having $\beta^{-1}+\beta^{-5}+\beta^{-6}$ as $\beta$-fractional part, under $\tau$. This means that, whereas there do not exist $x, y \in \mathbb{Z}_{\beta}$ such that $\{x+y\}_{\beta}=\beta^{-1}+\beta^{-5}+\beta^{-6}$, there exists a complex number, namely $\frac{2}{1-\alpha^{3}}$, which is the sum of two elements of $\mathcal{T}$ and admits an $\alpha$-expansion whose fractional part is of length 6 .

For any $x \in \operatorname{Fin}(\beta) \bigcap\left[0,1\left[\right.\right.$, let $d_{\beta}(x)=0 . v$ and $T_{. v}=\overline{\tau\left(\left\{y \in \operatorname{Fin}(\beta),\{y\}_{\beta}=x\right\}\right)}$. Due to Messaoudi [27], the fact that the complex numbers $\frac{2}{1-\alpha^{3}}$ and $\frac{1}{1-\alpha^{3}}$ both have three $\alpha$-expansions means that they belong to the fractal boundary of three different tiles $T_{. x}$. By computation, one gets $\left\{\frac{2}{1-\alpha^{3}}\right\} \subset \mathcal{T}_{\text {.100011 }} \cap \mathcal{T}_{\text {.001 }} \cap \mathcal{T}_{\text {.011 }}$ and $\left\{\frac{1}{1-\alpha^{3}}\right\} \subset \mathcal{T} \cap \mathcal{T}_{\text {.001 }} \cap \mathcal{T}_{\text {.011. }}$. Figure 4 gives a geometrical representation of these relations.

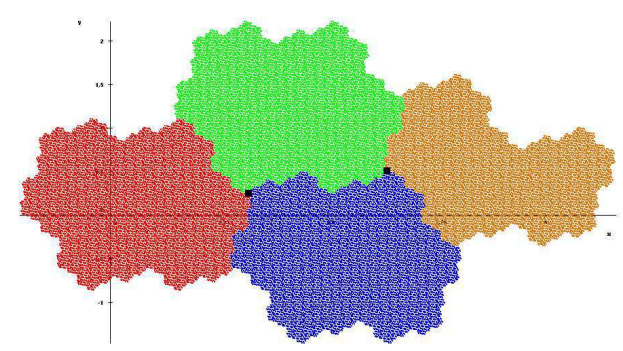

Fig. 4: Tiles $\mathcal{T}, \mathcal{T}_{.011}, \mathcal{T}_{\text {.001 }}$ and $\mathcal{T}_{\text {.100011 }}$

It would be interesting to compute the value of $L_{\odot}$ for the Tribonacci case. Due to [9], we know that $4 \leq L_{\odot} \leq 5$. Similarly as in the case of addition, one checks by pure computation that there exists only one real number $x$ such that $l_{f}(x)=5$, namely $x=\beta^{-1}+\beta^{-3}+\beta^{-5}$, which could be the $\beta$-fractional part of the product of two $\beta$-integers. We do not know whether there exist two $\beta$-integers $x$ and $y$ such that $\{x y\}_{\beta}=\beta^{-1}+\beta^{-3}+\beta^{-5}$; nevertheless we find that the complex numbers $\frac{1+\alpha}{1-\alpha^{3}}, \frac{1}{1-\alpha^{3}}$ and $\frac{\alpha^{2}}{1-\alpha^{3}}$ belong to $\mathcal{T}$ with $\frac{1+\alpha}{1-\alpha^{3}} \cdot \frac{1}{1-\alpha^{3}}=\frac{\alpha^{2}}{1-\alpha^{3}}+\alpha^{-1}+\alpha^{-3}+\alpha^{-5}$. Hence there exists an $\alpha$-expansion of $\frac{1+\alpha}{1-\alpha^{3}} \cdot \frac{1}{1-\alpha^{3}}$, the product of two elements which belong to $\mathcal{T}$, which admits an $\alpha$-fractional part whose fractional part is $\alpha^{-1}+\alpha^{-3}+\alpha^{-5}$. However, these informations are not sufficient to determine whether there exist $x, y \in \mathbb{Z}_{\beta}$ such that $\{x y\}_{\beta}=\beta^{-1}+\beta^{-3}+\beta^{-5}$. 


\section{Acknowledgements}

I am indebted to Valérie Berthé and Pierre Arnoux for their advices and corrections. I also thank Julien Cassaigne, Petr Ambrož, Zuzana Másáková, Edita Pelantová, Shigeki Akiyama and Anne Siegel for many interesting talks, remarks and ideas developed in this paper. I strongly thank the referees for their valuable comments and corrections.

\section{References}

[1] S. Akiyama. Pisot numbers and greedy algorithm. In Number theory (Eger, 1996), pages 9-21. de Gruyter, Berlin, 1998.

[2] S. Akiyama. Self affine tiling and Pisot numeration system. In Number theory and its applications (Kyoto, 1997), volume 2 of Dev. Math., pages 7-17. Kluwer Acad. Publ., Dordrecht, 1999.

[3] S. Akiyama. Cubic Pisot units with finite beta expansions. In Algebraic number theory and Diophantine analysis (Graz, 1998), pages 11-26. de Gruyter, Berlin, 2000.

[4] S. Akiyama. On the boundary of self affine tilings generated by Pisot numbers. J. Math. Soc. Japan, 54(2):283-308, 2002.

[5] S. Akiyama, F. Bassino, and C. Frougny. Automata for arithmetic Meyer sets. Lecture Notes in Computer Science, 2976:252-261, 2004.

[6] S. Akiyama and A. Pethő. On canonical number systems. Theoret. Comput. Sci., 270(12):921-933, 2002.

[7] S. Akiyama, H. Rao, and W. Steiner. A certain finiteness property of Pisot number systems. J. Number Theory, 107(1):135-160, 2004.

[8] S. Akiyama and J. M. Thuswaldner. A survey on topological properties of tiles related to number systems. Geom. Dedicata, 109:89-105, 2004.

[9] P. Ambrož, C. Frougny, Z. Másáková, and E. Pelantová. Arithmetics on number systems with irrational bases. Bull. Belgian Math. Soc. Simon Stevin, 10:641-659, 2003.

[10] D. Barache, B. Champagne, and J.-P. Gazeau. Pisot-cyclotomic quasilattices and their symmetry semigroups. In Quasicrystals and discrete geometry (Toronto, ON, 1995), volume 10 of Fields Inst. Monogr., pages 15-66. Amer. Math. Soc., Providence, RI, 1998.

[11] J. Bernat. Arithmetic automaton for Perron numbers. Accepté par Discrete Mathematics and Theoretical Computer Science, 2005.

[12] A. Bertrand. Développement en base de Pisot et répartition modulo 1. C. R. Acad. Sc., 385:419-421, 1977.

[13] Č. Burdík, C. Frougny, J. P. Gazeau, and R. Krejcar. Beta-integers as natural counting systems for quasicrystals. J. Phys. A, 31(30):6449-6472, 1998.

[14] V. Canterini. Connectedness of geometric representation of substitutions of Pisot type. Bull. Belg. Math. Soc. Simon Stevin, 10(1):77-89, 2003.

[15] V. Canterini and A. Siegel. Geometric representation of substitutions of Pisot type. Trans. Amer. Math. Soc., 353(12):5121-5144 (electronic), 2001.

[16] S. Fabre. Substitutions et $\beta$-systèmes de numération. Theoret. Comput. Sci., 137(2):219-236, 1995. 
[17] C. Frougny. Confluent linear numeration systems. Theoret. Comput. Sci., 106(2):183-219, 1992.

[18] C. Frougny. On-line addition in real base. In Mathematical foundations of computer science 1999 (Szklarska Porpolhk eba), volume 1672 of Lecture Notes in Comput. Sci., pages 1-11. Springer, Berlin, 1999.

[19] C. Frougny, Z. Masáková, and E. Pelantová. Complexity of infinite words associated with beta-expansions. Theor. Inform. Appl., 38(2):163-185, 2004.

[20] C. Frougny and B. Solomyak. Finite beta-expansions. Ergodic Theory Dynam. Systems, 12(4):713-723, 1992.

[21] J.-P. Gazeau and J.-L. Verger-Gaugry. Geometric study of the set of $\beta$-integers for a Perron number and mathematical quasicrystals. J. Théor. Nombres Bordeaux, 16:1-25, 2004.

[22] L.-S. Guimond, Z. Másáková, and E. Pelantová. Combinatorial properties of infinite words associated with cut-and-project sequences. J. Théor. Nombres Bordeaux, 15:697-725, 2003.

[23] L.-S. Guimond, Z. Másáková, and E. Pelantová. Arithmetics of beta-expansions. Acta Arith., 112(1):23-40, 2004.

[24] S. Ito and M. Kimura. On Rauzy fractal. Japan J. Indust. Appl. Math., 8(3):461-486, 1991.

[25] J. C. Lagarias. Geometric models for quasicrystals I. Delone sets of finite type. Discrete Comput. Geom., 21(2):161-191, 1999.

[26] M. Lothaire. Algebraic Combinatorics On Words. Cambridge University Press, 2002.

[27] A. Messaoudi. Frontière du fractal de Rauzy et système de numération complexe. Acta Arith., 95(3):195-224, 2000.

[28] Y. Meyer. Algebraic numbers and harmonic analysis. North-Holland Publishing Co., Amsterdam, 1972. North-Holland Mathematical Library, Vol. 2.

[29] Y. Meyer. Quasicrystals, Diophantine approximation and algebraic numbers. In Beyond quasicrystals (Les Houches, 1994), pages 3-16. Springer, Berlin, 1995.

[30] R. V. Moody. Meyer sets and their duals. In The mathematics of long-range aperiodic order (Waterloo, ON, 1995), volume 489 of NATO Adv. Sci. Inst. Ser. C Math. Phys. Sci., pages 403-441. Kluwer Acad. Publ., Dordrecht, 1997.

[31] W. Parry. On the $\beta$-expansions of real numbers. Acta Math. Acad. Sci. Hungar., 11:401-416, 1960.

[32] N. Pytheas Fogg. Substitutions in Dynamics, Arithmetics and Combinatorics (Berthé V., Ferenczi S., Mauduit C. and Siegel A.). Springer Lecture Notes in Mathematics, 1794, Berlin, 2002.

[33] G. Rauzy. Nombres algébriques et substitutions. Bull. Soc. Math. France, 110(2):147-178, 1982.

[34] G. Rauzy. Rotations sur les groupes, nombres algébriques et substitutions. Séminaire de théorie des nombres, 1987-1988.

[35] A. Rényi. Representations for real numbers and their ergodic properties. Acta Math. Acad. Sci. Hungar, 8:477-493, 1957.

[36] K. Scheicher and J. M. Thuswaldner. Canonical number systems, counting automata and fractals. Math. Proc. Cambridge Philos. Soc., 133(1):163-182, 2002. 
[37] K. Schmidt. On periodic expansions of Pisot numbers and Salem numbers. Bull. London Math. Soc., 12:269-278, 1980.

[38] D. Shechtman, I. Blech, D. Gratias, and J. Cahn. Metalic phase with with long-range orientational order and no translational symmetry. Phys. Rev. Lett., 53:1951-1953, 1984.

[39] V. F. Sirvent and Y. Wang. Self-affine tiling via substitution dynamical systems and Rauzy fractals. Pacific J. Math., 206(2):465-485, 2002.

[40] B. Solomyak. Substitutions, adic transformations, and beta-expansions. In Symbolic dynamics and its applications (New Haven, CT, 1991), volume 135 of Contemp. Math., pages 361-372. Amer. Math. Soc., Providence, RI, 1992.

[41] W. P. Thurston. Groups, tilings and finite state automata. Summer 1989 AMS Colloquium lectures, 1989. 
